\title{
Dense gas and Star Formation: Characteristics of Cloud Cores Associated with Water Masers.
}

\author{
René Plume, D.T. Jaffe, Neal J. Evans II \\ Department of Astronomy, University of Texas at Austin \\ J. Martín-Pintado, and J. Gómez-González \\ Centro Astronomico de Yebes
}

\begin{abstract}
We have observed 150 regions of massive star formation, selected originally by the presence of an $\mathrm{H}_{2} \mathrm{O}$ maser, in the $\mathrm{J}=5 \rightarrow 4,3 \rightarrow 2$, and $2 \rightarrow 1$ transitions of $\mathrm{CS}$, and 49 regions in the same transitions of $\mathrm{C}^{34} \mathrm{~S}$. Over $90 \%$ of the 150 regions were detected in the $\mathrm{J}=2 \rightarrow 1$ and $3 \rightarrow 2$ transitions of $\mathrm{CS}$ and $75 \%$ were detected in the $\mathrm{J}=5 \rightarrow 4$ transition. We have combined the data with the $\mathrm{J}=7 \rightarrow 6$ data from our original survey (Plume et al. 1992) to determine the density by analyzing the excitation of the rotational levels. Using Large Velocity Gradient (LVG) models, we have determined densities and column densities for 71 of these regions. The gas densities are very high $(\langle\log (\mathrm{n})\rangle=5.9)$, but much less than the critical density of the $\mathrm{J}=7 \rightarrow 6$ line. Small maps of 25 of the sources in the $\mathrm{J}=5 \rightarrow 4$ line yield a mean diameter of 1.0 pc. Several estimates of the mass of dense gas were made for the sources for which we had sufficient information. The mean virial mass is $3800 \mathrm{M}_{\odot}$. The mean ratio of bolometric luminosity to virial mass $(L / M)$ is 190, about 50 times higher than estimates using $\mathrm{CO}$ emission, suggesting that star formation is much more efficient in the dense gas probed in this study. The gas depletion time for the dense gas is roughly $1.3 \times 10^{7} \mathrm{yr}$, comparable to the timescale for gas dispersal around open clusters and $\mathrm{OB}$ associations. We find no statistically significant linewidth-size or density-size relationships in our data. Instead, both linewidth and density are larger for a given size than would be predicted by the usual relationships. We find that the linewidth increases with density, the opposite of what would be predicted by the usual arguments. We estimate that the luminosity of our Galaxy (excluding the inner $400 \mathrm{pc}$ ) in the CS J $=5 \rightarrow 4$ transition is 15 to 23 $\mathrm{L}_{\odot}$, considerably less than the luminosity in this line within the central 100 pc of NGC 253 and M82. In addition, the ratio of far-infrared luminosity to CS luminosity is higher in M82 than in any cloud in our sample.
\end{abstract}

Subject headings: ISM:molecules — Stars:formation

\section{Introduction}

Very dense gas $\left(\mathrm{n} \geq 10^{5} \mathrm{~cm}^{-3}\right)$ has an important effect upon star formation in molecular clouds. The presence of very dense gas affects the Jeans mass and other measures of stability. In addition, the quantity of very dense gas has consequences for the calculated star formation efficiency, since it is this material that actively participates in star formation (Lada et al. 1991; Solomon, Radford, \& Downes 1990). Most stars (even low-mass stars) form in regions where high-mass stars are forming (Elmegreen 1985). In high-mass 
star-forming regions, winds and radiation from nearby, newly-formed stars can disrupt the local gas and effectively shut down further star formation. Cores of very dense gas, however, resist these disruptive forces and can help to maintain star formation in the hostile environments associated with young, massive stars (Klein et al. 1983; LaRosa 1983).

How universal is very dense gas $\left(\mathrm{n} \geq 10^{5} \mathrm{~cm}^{-3}\right)$ ? Benson \& Myers (1989) and Zhou et al. (1989) have shown that densities of order $10^{4}$ to $10^{5} \mathrm{~cm}^{-3}$ are common in low-mass star-forming regions. Studies of a few selected regions that are forming massive stars (e.g., Jaffe et al. 1983; Cunningham et al. 1984; Snell et al. 1984; Richardson et al. 1985; Mundy et al. 1987; Mezger et al. 1988; Churchwell, Walmsley, \& Wood 1992; Wang et al. 1993; Bergin et al. 1996; Hofner et al. 1996) have demonstrated the presence very dense gas $\left(\mathrm{n}=10^{5}\right.$ to $\left.10^{6} \mathrm{~cm}^{-3}\right)$. However, it is unclear whether such gas is common to all regions forming massive stars. The overall sample of such regions is small, and the studies used a variety of selection criteria and density measurement techniques.

To assess the prevalence of very dense gas, we need to determine densities using a consistent method in a large and representative sample of regions forming massive stars. The small beam sizes at frequencies used to probe for very dense gas, along with limited amounts of available telescope time, make it impossible to map completely all the regions known to be forming massive stars. Therefore, we require a pointer to likely locations of active star formation within molecular clouds. $\mathrm{H}_{2} \mathrm{O}$ masers are ideal for this purpose since they contain at least small amounts of extremely dense gas $\left(\mathrm{n}>10^{10} \mathrm{~cm}^{-3}\right)$ (Elitzur et al. 1989; Strelnitskij 1984); and, in well-studied regions, they are intimately associated with star formation (e.g., Genzel \& Downes 1977, 1979; Jaffe et al. 1981; Wood \& Churchwell 1989; Churchwell 1990).

Our initial survey (Plume et al. 1992; hereafter Paper I) searched for thermal emission from dense gas associated with $\mathrm{H}_{2} \mathrm{O}$ masers. The sample consisted of 179 of the $181 \mathrm{H}_{2} \mathrm{O}$ masers listed in the catalog of Cesaroni et al. (1988) as "HII region" (i.e., not a late-type star) masers which were north of $\delta=-30^{\circ}$ and had positions known to better than $8^{\prime \prime}$. We used the $\mathrm{J}=7 \rightarrow 6$ transition of CS, which has a high critical density $\left(\mathrm{n}_{\text {crit }} \approx 2 \times 10^{7} \mathrm{~cm}^{-3}\right)$, selecting regions of very dense gas.

Observations of a single transition of the CS molecule are not sufficient to determine gas density. Therefore, we have observed the $\mathrm{J}=5 \rightarrow 4,3 \rightarrow 2$, and $2 \rightarrow 1$ transitions of CS in 150 of the 179 regions from our initial CS J $=7 \rightarrow 6$ survey (Paper I). Of the regions sampled in the current survey, 85 (57\%) contained CS $7 \rightarrow 6$ emission; this percentage is similar to that of the original CS $7 \rightarrow 6$ survey (104 of 179 , or $58 \%$; Paper I), indicating that this study was not biased towards the densest regions. We also observed the same transitions of $\mathrm{C}^{34} \mathrm{~S}$ in 49 of the strongest $\mathrm{CS}$ sources. The $\mathrm{C}^{34} \mathrm{~S}$ data yield an independent measurement of the densities. We have also mapped 21 sources in the $\mathrm{J}=5 \rightarrow 4$ line of CS to determine the size of the cores.

In $\S 3$, we present basic detection statistics and discuss the individual spectra and the maps. In $\S 4$, we present densities and column densities based upon excitation analysis of the data, consider effects of opacity and temperature uncertainties on the results, and compute masses. In $\S 5$, we consider issues like the star formation efficiency, compare these regions to other regions, and estimate the luminosity of the galaxy in the $\mathrm{CS} \mathrm{J}=5 \rightarrow 4$ line.

\section{Observations}

We observed the $\mathrm{J}=5 \rightarrow 4,3 \rightarrow 2$ and $2 \rightarrow 1$ transitions of CS and $\mathrm{C}^{34} \mathrm{~S}$ in 1990 June, 1991 April, and 1991 October at the IRAM 30-m telescope at Pico Veleta, Spain, with the 3-mm, 2-mm, and 1.3-mm SIS 
receivers tuned to single-sideband mode. Table 1 lists the line frequencies, main beam efficiencies $\left(\eta_{m b}\right)$, beamsizes, typical system temperatures, and velocity resolutions for each transition. To convert to the $\mathrm{T}_{R}^{*}$ scale (Kutner and Ulich 1981), the data were scaled by $\eta_{m b}$ (i.e., $\mathrm{T}_{R}^{*}=\mathrm{T}_{A}^{*} / \eta_{m b}$ ). In IRAM notation, $\eta_{m b}=B_{\text {eff }} / F_{\text {eff }}$, the back spillover and scattering efficiency divided by the forward spillover and scattering efficiency. For all excitation calculations we have assumed that the source is fully resolved, so that $\mathrm{T}_{R}^{*}=$ $\mathrm{T}_{R}$, the Rayleigh-Jeans temperature of a spatially resolved source observed with a perfect telescope above the atmosphere.

The pointing was checked regularly. In 1990 June, we pointed on continuum emission from K3-50, $\mathrm{W} 3(\mathrm{OH})$, and Jupiter and found the pointing to be very sensitive to changes in azimuth and elevation, with a maximum spread of $\approx 15^{\prime \prime}$. Consequently, we made nine-point maps on a $16^{\prime \prime}$ grid for each source, while defining a pointing curve. The reliability of these observations will be discussed below. The pointing curves were well-determined by the time we made the $\mathrm{C}^{34} \mathrm{~S}$ observations. In $1991 \mathrm{April}$, we checked the pointing with continuum observations of W3(OH), NGC 7027, BL Lac, and Saturn. For this observing run, the telescope pointing was accurate to within $4^{\prime \prime} \mathrm{rms}$. In 1991 October, the pointing was good to $5^{\prime \prime}$ rms.

The data were calibrated to the $\mathrm{T}_{A}^{*}$ scale using the chopper wheel method (Penzias \& Burrus 1973). To include the effects of different sideband gains, we observed the line calibration sources W51M, W51N, W44, W3 $(\mathrm{OH})$, and DR21S in 1990 June, and IRC+10216, W3(OH), and Orion IRc2 in 1991 April. These sources were observed by Mauersberger et al. (1989) in single-sideband mode with an image-sideband rejection of $>8 \mathrm{~dB}$. We compared our observed antenna temperatures with those tabulated by Mauersberger et al. (1989) and adjusted our temperature scale to agree with theirs. For the 1990 June run, we did not need to adjust the CS J $=2 \rightarrow 1$ data. The June CS $3 \rightarrow 2$ and $5 \rightarrow 4$ transitions needed to be reduced in strength by $\approx 10 \%$. The 1991 April CS $\mathrm{J}=2 \rightarrow 1$ and $5 \rightarrow 4$ data both needed to be increased by $20 \%$. The 1991 April $\mathrm{CS} \mathrm{J}=3 \rightarrow 2$ data were multiplied by a factor of 2 and assigned a $50 \%$ calibration error. Lacking a standard source for the $\mathrm{C}^{34} \mathrm{~S} \mathrm{~J}=3 \rightarrow 2$ line, we also scaled it up by a factor of 2 and assigned a $50 \%$ calibration error. In 1991 October, no scaling was necessary. We have also compared the CS $\mathrm{J}=7 \rightarrow 6$ results of Plume et al. (1991) to the more recent single-sideband observations of Wang et al. (1994). For the five sources in common, the ratio of $\mathrm{T}_{A}^{*}$ values is $0.98 \pm 0.19$, indicating that the calibration of the $\mathrm{J}=7 \rightarrow 6$ data in Paper I was good.

We also used the CSO for some auxiliary observations, with parameters shown in the last 4 lines of Table 1 . We made small maps of the CS $\mathrm{J}=5 \rightarrow 4$ transition toward 21 of the sources in our sample in 1994 June. The pointing was checked on planets and repeated to $3^{\prime \prime}$. We observed the $\mathrm{J}=7 \rightarrow 6$ transition of $\mathrm{C}^{34} \mathrm{~S}$ in 1991 June. Beam sizes and efficiencies are based on Mangum (1993). For a few sources, we also observed the $\mathrm{J}=10 \rightarrow 9$ transition of CS in 1993 December. The pointing was checked on planets and found to be constant to $6^{\prime \prime}$. The beamsize was assumed to be $14^{\prime \prime}$, based on scaling from lower frequencies, and the efficiency was measured on Saturn. Finally, the $J=14 \rightarrow 13$ line of CS was observed toward two sources in 1994 December. The pointing was checked by observing planets and was constant to $6{ }^{\prime \prime}$. The beamsize was assumed to be $11^{\prime \prime}$. The optical depth at the zenith was measured by tipping to be 0.35 at $685 \mathrm{GHz}$. For all the CSO observations, two AOS spectrometers were used, with resolutions of about 140 $\mathrm{kHz}$ and $1 \mathrm{MHz}$. The choice of spectrometer for determining line parameters was made on the basis of getting sufficient resolution and signal-to-noise in the line.

\section{Results}




\subsection{Detection Statistics}

Table 2 lists the $150 \mathrm{H}_{2} \mathrm{O}$ maser sites observed at IRAM in the CS J $=5 \rightarrow 4,3 \rightarrow 2$, and $2 \rightarrow 1$ transitions. Tables 1 and 2 of Paper I list the positions of the masers and the CS J=7 $\rightarrow 6$ line parameters or upper limits. Table 2 of the present paper lists the source names in order of increasing galactic longitude, the radiation temperature $\left(\mathrm{T}_{R}^{*}\right)$, integrated intensity $\left(\int T_{R}^{*} d v\right)$, velocity centroid $\left(\mathrm{V}_{L S R}\right)$, and full width at half maximum (FWHM) for the three transitions. For CS data obtained in 1990 June, we list the line parameters at the position in the nine-point map with the strongest emission in the $\mathrm{J}=5 \rightarrow 4$ line. This choice is based on the results of $\S 3.2$, where we find that the $\mathrm{J}=5 \rightarrow 4$ emission almost always peaks at the maser position. While the line parameters for 1990 June are useful in detection statistics and as a guide for follow-up work, we have found that the position correction was inadequate for them to be used together with the $\mathrm{J}=7 \rightarrow 6$ data to determine densities; therefore we do not use the 1990 June data in $\S 4$. For undetected lines, the upper limits to $\mathrm{T}_{R}^{*}$ are $3 \sigma$. For CS $\mathrm{J}=3 \rightarrow 2$ and $2 \rightarrow 1$, we have tabulated only the data with the highest spectral resolution. We also observed the $\mathrm{C}^{34} \mathrm{~S}$ lines in 49 of the strongest $\mathrm{CS}$ emitters. The results for $\mathrm{C}^{34} \mathrm{~S}$ are presented in Table 3 . Transitions listed with dashes $(-)$ instead of values or upper limits to $\mathrm{T}_{R}^{*}$ were not observed. Table 4 has the results for $\mathrm{J}=10 \rightarrow 9$ and $14 \rightarrow 13$.

Usually, we obtained the line parameters from Gaussian fits to the lines but some sources listed in Table 2 had spectra with more than one peak. To determine the line parameters in these cases, we took the following approach. First, if the profiles of the higher $\mathrm{J}$ (i.e., $7 \rightarrow 6$ or $5 \rightarrow 4$ ) lines or $\mathrm{C}^{34} \mathrm{~S}$ lines (where available) matched one or more of the peaks seen in the lower $\mathrm{J}$ transitions, we assumed that the source was composed of distinct cloud components (e.g., Figure 1a); and we derived the line parameters by performing a multiple Gaussian fit to the whole profile. Each Gaussian component is listed individually in Table 2. Three sources have 2 velocity components and one has 3 components; these are identified in Tables 2 and 3 by the notation "C\#" (where \# is the component number). With the inclusion of all the separate components, Table 2 displays results for 155 cloud components. Second, if comparison of CS data with $\mathrm{C}^{34} \mathrm{~S}$ data indicated that the CS line was self-absorbed (Figure 1b shows an example of this situation), we calculated the line parameters $\left(\int T_{R}^{*} d V, \mathrm{~V}_{L S R}\right.$, and FWHM) from moment integrals over the profile. Then $T_{R}^{*}$ was calculated from $\int T_{R}^{*} d V /$ FWHM (values given in parentheses in Table 2). Only 18 of the 150 spectra were obviously self-absorbed in CS $2 \rightarrow 1$, with smaller numbers showing obvious self-absorption in the higher-J lines. Of course, self-absorption may exist at a less obvious level in other sources.

Figure 2 illustrates the detection rate for the observed CS transitions. The distribution counts as detected only those sources with observed $\mathrm{T}_{R}^{*} \geq 0.5 \mathrm{~K}$. Because the sensitivity achieved for the CS J = $7 \rightarrow 6$ line (Paper I) was similar to that for the lower $\mathrm{J}$ transitions, the drop in the detection rate towards higher rotational levels reflects a real drop in the number of sources exhibiting emission at the same level in the higher J lines.

\subsection{Extent of the Dense Gas: CS J $=5 \rightarrow 4$ Maps}

To determine the effect that very dense gas has upon star formation, we need to know the extent of the gas and its location within the star-forming regions. We have observed 21 of our sources in the CS $5 \rightarrow 4$ line with the CSO. For each source, we made a cross-scan in R.A. and Dec., typically consisting of 9 points. For most of the sources, the separation of the observed points was $30^{\prime \prime}$. For a few of the smaller sources, 
we made the observations at $15^{\prime \prime}$ intervals. In addition, we have assembled from the literature data taken with the same equipment for four other sources from our survey. Table 5 lists the mapping results for all 25 sources. The integrated intensities listed in Table 5 are for the interpolated maximum along each cross scan. From the maps we derived diameters and beam correction factors, $F_{c}=\left(\Omega_{\text {source }}+\Omega_{\text {beam }}\right) / \Omega_{\text {beam }}$. The beam correction factors were calculated assuming that a Gaussian was a good representation of both the beam shape and the source intensity distribution. Using the integrated intensity, the $F_{c}$, and the distances, $d(\mathrm{kpc})$, we calculated the luminosity in the CS $\mathrm{J}=5 \rightarrow 4$ line from

$$
L(\mathrm{CS} 5-4)=1.05 \times 10^{-5} L_{\odot} d^{2} F_{c} \int T_{R}^{*} d v .
$$

Table 5 also lists the offsets of the CS $5 \rightarrow 4$ peaks from the maser positions in arcseconds. With the exception of a few of the larger sources, differences in the peak position of the CS $5 \rightarrow 4$ distribution and the $\mathrm{H}_{2} \mathrm{O}$ maser position are smaller than the combined pointing uncertainties and maser positional uncertainties ( $\pm 3^{\prime \prime}$ and $\leq \pm 8^{\prime \prime}$, respectively). Jenness et al. (1995) have also found a very good correlation between the peak of the submillimeter emission and the maser position. The mean diameter of the sources listed in Table 3 is $1.0 \mathrm{pc}$. The dispersion about this mean, however, is large $(0.7 \mathrm{pc})$. If one examines sources at $d \leq 3.0 \mathrm{pc}$, the mean diameter is $0.5 \mathrm{pc}$ with a dispersion of $0.4 \mathrm{pc}$. This difference, while significant, probably does not arise from observational biases in the CS data. Most of the more distant sources are well resolved and bright. It is more likely that the differences arise from selection biases in the original samples used to search for $\mathrm{H}_{2} \mathrm{O}$ masers. Complete mapping of the CS $5 \rightarrow 4$ line in several sources gives similar sizes. The emission in NGC2024 has a diameter of 0.4 pc, while S140 has a diameter of 0.8 pc (Snell et al. 1984). The emission in M17 is more extensive: $2.3 \mathrm{pc}$ in $5 \rightarrow 4$ (Snell et al.); $2.1 \mathrm{pc}$ in $7 \rightarrow 6$ (Wang et al. 1993).

\section{Analysis}

With the addition of the lower $\mathrm{J}$ transitions in the present study to the CS $\mathrm{J}=7 \rightarrow 6$ data from Paper I, we can determine densities in a large sample of star-forming regions. In $\S 4.1$, we discuss the calculations and examine the effects of opacity and uncertainties in kinetic temperature on density and column density determinations. In $\S 4.2$, we consider the effects of density inhomogeneities, and we compute masses in $\S 4.3$.

\subsection{Densities and Column Densities}

To determine densities and column densities, we used a large velocity gradient (LVG) code to solve the coupled equations of statistical equilibrium and radiative transfer, including the first 20 rotational levels of CS in the calculation. We assume that the gas has a constant density and temperature and that it uniformly fills all the beams used in this study. We calculated a $20 \times 20$ grid of radiation temperatures in column density per velocity interval - density space for a kinetic temperature of $50 \mathrm{~K}$. The CS densities in the LVG model grid ran from $10^{4}$ to $10^{8} \mathrm{~cm}^{-3}$, and the column densities per velocity interval $(\mathrm{N} / \Delta \mathrm{v})$ ranged from $10^{11}$ to $10^{16} \mathrm{~cm}^{-2} / \mathrm{km} \mathrm{s}^{-1}$. These ranges span the parameter space of all solutions which fit our data. All the models converged to a solution. 
Using a $\chi^{2}$ minimization routine, we fit the LVG models to the observed CS line intensities. Table 6 lists the densities for 71 sources. We have not included fits for the CS data obtained in 1990 June, for reasons discussed below. We have listed the log of the density and column density, along with the value of $\chi^{2}$ and a key to which transitions were used and whether the lines were self-absorbed. The values of density and column density apply to the region selected by the typical beam used for the observations (about $20^{\prime \prime}$ ). The $\chi^{2}$ values allow us to assess whether the models (any of the points in the LVG grid) are a good representation of the data. The distribution of $\chi^{2}$ values for sources with 4 transitions (40 sources) is similar to what is expected theoretically if the model is a reasonable fit to the data, as is the distribution for sources with only three transitions (31 sources). These facts suggest that our estimates of the calibration uncertainties are reasonable. We originally included the 1990 June data in the fits, but they had a very high percentage of bad fits, leading us to conclude that the uncertain pointing made them unsuitable for combining with the CSO $\mathrm{J}=7 \rightarrow 6$ data. The 8 self-absorbed sources with fits in Table 6 (marked by a flag) do not have $\chi^{2}$ significantly worse than the other sources. One source with 3 transitions (212.25-1.10) produced a very uncertain density, and we have excluded it from the statistics that follow.

The mean logarithmic density for sources with detected emission from all 4 CS transitions is $\langle\log (\mathrm{n})\rangle=5.93 \pm 0.23$, where 0.23 represents the standard deviation of the distribution. The mean logarithmic column density is $\langle\log (\mathrm{N})\rangle=14.42 \pm 0.49$. The results for the sources undetected in $\mathrm{J}=7 \rightarrow 6$ are $\langle\log (\mathrm{n})\rangle=5.59 \pm 0.39 ;\langle\log (\mathrm{N})\rangle=13.57 \pm 0.35$. Figure 3 shows histograms of the densities and column densities. The solid line plots the densities determined from all 4 CS transitions and the dashed line is the density distribution for sources without $\mathrm{J}=7 \rightarrow 6$ detections. These results show that the difference between a CS $7 \rightarrow 6$ detection and non-detection is more related to column density than to volume density. Therefore, the detectability of lines of high critical density is more affected by the quantity of dense gas present than by its density. To check whether the difference was solely a result of having a $\mathrm{J}=7 \rightarrow 6$ line to fit, we re-fit the sources with $7 \rightarrow 6$ detections, forcing the $\chi^{2}$ fitting routine to ignore the CS $7 \rightarrow 6$ line and to fit only the 3 lower transitions. The resulting $\langle\log (\mathrm{n})\rangle$ is $5.71 \pm 0.19$, and $\langle\log (\mathrm{N})\rangle$ is $14.36 \pm 0.49$. This result confirms our conclusion that the most significant difference between a $\mathrm{J}=7 \rightarrow 6$ detection and a non-detection is the column density.

What effect would high opacity in the CS lines have on the derived densities and column densities? Eighteen of the sources in this survey have noticeable self-absorption in at least one transition. In addition, an LVG model run for the mean density, column density, and linewidth results in CS line opacities that are roughly unity. Thus, self-absorption may affect the fits, even if it is not apparent in the line profiles. Since the $\mathrm{C}^{34} \mathrm{~S}$ transitions will usually be optically thin, we independently fit the $\mathrm{C}^{34} \mathrm{~S}$ transitions to an LVG model grid, with a range of parameters identical to those used in the original CS grid. Table 6 lists the densities, column densities, and $\chi^{2}$ derived from fits to the $\mathrm{C}^{34} \mathrm{~S}$ data. Problems with the receivers during the $\mathrm{C}^{34} \mathrm{~S}$ observations meant that we have various combinations of lines to fit, as indicated by the key in Table 6. There are few sources with both adequate $\mathrm{CS}$ and acceptable $\mathrm{C}^{34} \mathrm{~S}$ data. The fits to the sources with three transitions of $\mathrm{C}^{34} \mathrm{~S}$ give $\langle\log (\mathrm{n})\rangle=5.95 \pm 0.20$, essentially identical to the $\langle\log (\mathrm{n})\rangle$ derived from 4 transitions of CS. The mean difference between $\mathrm{CS}$ and $\mathrm{C}^{34} \mathrm{~S}$ in $\log (\mathrm{n})$ is $0.07 \pm 0.24$, indicating no significant difference in the derived densities. It is unlikely that the densities calculated for sources in our survey from the CS lines alone are seriously affected by CS optical depth. The average isotope ratio, $N(C S) / N\left(C^{34} S\right)$, is $5.1 \pm 2.2$, clearly less than the terrestrial ratio, and lower than the isotope ratios of 9-17 found by Mundy et al. (1986) and 13 (Wang et al. 1993). Chin et al. (1996) have recently found evidence for low values of this ratio in the inner Galaxy, but our values are lower still. It is likely that our procedure has underestimated $N(C S)$ to some extent. For this reason, and also because these ratios are not very well determined for individual sources, we have adopted an isotopic abundance ratio of 10 in what 
follows.

By increasing the number of transitions, simultaneous fitting of the $\mathrm{CS}$ and $\mathrm{C}^{34} \mathrm{~S}$ data should, in principle, allow us to determine the densities and column densities more accurately. Using the LVG model grid for CS and constraining the isotope ratio to be 10 , we fit $\mathrm{CS}$ and $\mathrm{C}^{34} \mathrm{~S}$ transitions simultaneously. The results are listed in Table 6 . While neither the densities nor the column densities are significantly different from those determined from fits to the CS data alone, $\chi^{2}$ is considerably larger. The poor fits probably result from assuming a fixed isotopic abundance ratio for all sources.

It is likely that many of the regions of massive star formation contained within this study have temperatures in excess of $50 \mathrm{~K}$. At the densities implied by the CS observations, the gas kinetic temperature will be coupled to the dust temperature. For grains with opacity decreasing linearly with wavelength, one can write

$$
T_{D}=C\left[\frac{L}{\theta^{2} d^{2}}\right]^{0.2},
$$

where $L$ is the luminosity in solar units, $d$ is the distance in kpc, and $\theta$ is the angular separation from the heating source in arcseconds. Using these units, $C=15$ to 40 (Makinen et al. 1985, Butner et al. 1990). We can estimate the range of temperatures in our sources from the luminosities in Table 7 and distances in Table $5 ;\left\langle\left(L / d^{2}\right)^{0.2}\right\rangle=7.5 \pm 1.6$. At a radius of $10^{\prime \prime}$, characteristic of the beams in Table 1 and the beam of the $\mathrm{J}=7 \rightarrow 6$ observations, $T_{D}=50$ to $100 \mathrm{~K}$. To assess the effects of temperature uncertainties on the derived source properties, we also fit the sources with 4 transitions to a grid of models run for a temperature of $100 \mathrm{~K}$. The value of $\langle\log (\mathrm{n})\rangle$ decreased by 0.3 and the value of $\langle\log (\mathrm{N})\rangle$ was essentially unchanged. Regardless of the assumed temperature, our data imply a thermal pressure, $n T \sim 4 \times 10^{7} \mathrm{~K}$ $\mathrm{cm}^{-3}$, which is much higher than found in regions not forming massive stars.

Within the limitations of a single-density model, we conclude that the effects of opacity and temperature on the determinations of density are not severe (about at the factor of 2 level). Typical densities in regions detected in the $\mathrm{J}=7 \rightarrow 6$ survey are $10^{6} \mathrm{~cm}^{-3}$. Toward water masers not detected in the $\mathrm{J}=7 \rightarrow 6$ survey, the densities are about a factor of 2 less, but the column densities of CS are about a factor of 7 less, on average, than the values found for regions detected in the $\mathrm{J}=7 \rightarrow 6$ line. The densities for both groups of sources are considerably less than the critical density of the CS $\mathrm{J}=7 \rightarrow 6$ line $\left(2 \times 10^{7} \mathrm{~cm}^{-3}\right)$, reminding us that detection of emission from a hard-to-excite line does not imply the existence of gas at the critical density. Molecules can emit significantly in high-J transitions with critical densities considerably above the actual density because of trapping and multilevel effects (see also Evans 1989). For example, levels with $\mathrm{J}>>0$ have many possible routes for excitation by collisions, but only one radiative decay path.

The high densities found in this survey of regions forming massive stars are similar to those obtained from other, more detailed, studies of individual, luminous, star-forming regions (see ref. in $§ 1$ ). Consequently, the results found from studies of a few clouds can be applied, in a statistical sense, to the broader sample of massive star-forming regions.

\subsection{Multiple Density Models}

Our LVG analysis assumes that the density is uniform and that the emitting gas fills the beam. How good are these assumptions? Figure 4 gives examples of LVG model fits to several of the sources: three with

good fits and three with bad fits, as measured by the $\chi^{2}$ value. While the LVG models generally fit the data 
within the uncertainties, a closer look reveals that the discrepancies between model and observation are very consistent, even for the good fits. Almost all fits overpredict the $3 \rightarrow 2$ and $5 \rightarrow 4$ lines and underpredict the $2 \rightarrow 1$ and $7 \rightarrow 6$ lines. Thus, the data have, on average, a smaller variation of intensity with $J$ than do the best-fit LVG models, as would be expected for a source with a mixture of gas at different densities. In this section, we examine models with varying densities to see how well they explain the intensity versus J distribution.

Snell et al. (1984) and Wang et al. (1993) have discussed the effects of fitting a single density to the CS emission from a mixture of gas at about $10^{6} \mathrm{~cm}^{-3}$ and gas lower in density by about a factor of 10 . They showed that, until the filling factor of the high density gas becomes very small (i.e., $f<0.2)$, the density derived from fitting a single density model matches that of the high density component to within a factor of two. The CS transitions we have observed should behave in a similar way in that they are biased toward measuring gas with densities close to $10^{6} \mathrm{~cm}^{-3}$.

We now ask a more radical question. Could the apparent density near $10^{6} \mathrm{~cm}^{-3}$ be an artifact of fitting to a single density a mixture of ultra-dense gas $\left(\mathrm{n}=10^{8} \mathrm{~cm}^{-3}\right)$ and gas at a much lower $\left(\mathrm{n}=10^{4} \mathrm{~cm}^{-3}\right)$ density? In this picture, the histogram of densities (Figure 3) would be produced by varying the filling factor of the dense component. We chose a value of $10^{8} \mathrm{~cm}^{-3}$ for the density of the ultra-dense gas because the $7 \rightarrow 6$ transition becomes completely thermalized at that density. Thus, the component with $\mathrm{n}=10^{8}$ $\mathrm{cm}^{-3}$ represents any gas with $\mathrm{n} \geq 10^{8} \mathrm{~cm}^{-3}$. We synthesized clouds from a mixture of these two components at 20 values of $\mathrm{N} / \Delta \mathrm{v}$ between $10^{12}$ and $10^{16} \mathrm{~cm}^{-2} / \mathrm{km} \mathrm{s}^{-1}$. For each density and column density, we used the LVG code to calculate the expected emission. We then varied the filling factor of the ultra-dense gas $(f)$ and the low-density gas $(1-f)$, with $0 \leq f \leq 1$ in steps of 0.05 , and summed the contributions to each transition for each possible combination of $f$, column density of the gas at $\mathrm{n}=10^{4} \mathrm{~cm}^{-3}\left(\mathrm{~N}_{\text {low }}\right)$, and column density of the gas at $n=10^{8} \mathrm{~cm}^{-3}\left(\mathrm{~N}_{\text {high }}\right)$. These results then formed a grid of models which could be fitted to the data, just as the single-density models had been fitted. We found that the $\chi^{2}$ value worsened, despite the extra free parameter, for sources where the single-density fit had been good $\left(\chi^{2} \leq 1\right)$. On the other hand, the sources which were poorly fitted $\left(\chi^{2}>1\right)$ with the single-density model were better fitted with the two-density model. The two-density fits typically required very high column densities $(\langle\log (\mathrm{N})\rangle=16.16)$ of the low-density gas compared to those of the ultra-dense gas $(\langle\log (\mathrm{N})\rangle=13.85)$.

To see if we could constrain the amount of ultra-dense gas in the sources with poor single-density fits, we followed a similar, but less restrictive, procedure. We started by assuming that the CS J $=2 \rightarrow 1$ and $3 \rightarrow 2$ transitions effectively probe the low density gas in the beam, and we used them to fit the density ( $\mathrm{n}_{\text {low }}$ ) and column density $\left(\mathrm{N}_{\text {low }}\right)$ of the low-density component. We then used the LVG code to obtain the expected emission from each rotational transition for a gas at this density and column density at a temperature of $50 \mathrm{~K}$. These intensities, multiplied by $(1-f)$, were used to represent the lower density component. We then searched a parameter space of $f$ and $\log (\mathrm{N} / \Delta \mathrm{v})$ for the best values for the ultra-dense component (density once again fixed at $\left.10^{8} \mathrm{~cm}^{-3}\right)$. We summed $(1-f)$ times the lower density intensities and $f$ times the ultra-dense gas intensities and compared this sum to the observations. This method has a large number of free parameters: $f, \mathrm{n}_{\text {low }}, \mathrm{N}_{\text {low }} / \Delta \mathrm{v}$, and $\mathrm{N}_{\text {high }} / \Delta \mathrm{v}$, which are constrained by only 4 transitions. Furthermore, it does not correct the properties of the lower density component for the contributions of the high density gas to the $\mathrm{J}=2 \rightarrow 1$ and $3 \rightarrow 2$ emission. We use it for illustrative purposes only. We show the two-density fits as dashed lines in Figure 4, but we do not tabulate the results. The mean properties of these solutions for the sources with single-density $\chi^{2}>1$ are as follows: $f=0.22, \log \left(\mathrm{n}_{\text {low }}\right)=5.4 \pm 0.3, \log \left(\mathrm{N}_{\text {low }}\right)=14.39$, and $\log \left(\mathrm{N}_{\text {high }}\right)=14.39$ (equal column densities in the two components). Thus, in general, the filling factor of ultra-dense gas is small (less than 25\%), and the data still favor a large amount of gas at $n>10^{5} \mathrm{~cm}^{-3}$. 
Another possible source model is a continous density gradient, such as a power law. Power-law density distributions have been proposed for regions of low-mass star formation on theoretical grounds (Shu 1977) and seem to fit the observations well in some cases (e.g., Zhou et al. 1991). They have also been applied to some regions forming stars of higher mass (e.g., Zhou et al. 1994; Carr et al. 1995). The latter reference is particularly relevant here, as it included a more complete analysis of GL2591 (called CRL2591 in this paper), including data from this paper, but adding other data. While Table 6 indicates a good fit to the data for that source with a single-density model, Carr et al. found that a single density cannot fit all the data, when other data are included, particularly $\mathrm{J}=5 \rightarrow 4$ and $10 \rightarrow 9$ data from the CSO. They developed models with power-law density and temperature gradients that fit all the data. We can use the example of CRL2591 to explore the meaning of the densities in Table 6 if the actual density distribution is a power law. If $n(r)=n_{1} r_{p c}^{-\alpha}$, with $n_{1}$ (the density at $1 \mathrm{pc}$ ) set by matching the line profiles (Carr et al. 1995), the density in Table 6 is reached at radii of $18^{\prime \prime}$ to $7^{\prime \prime}$ for $1 \leq \alpha \leq 2$, corresponding to filling factors of 0.3 to 0.6 in our largest beam. We conclude that, in this source, the densities derived in this study characterize gas on scales somewhat smaller than our beams, if the source has a density gradient. Similar studies of other sources are needed to see if this conclusion can be generalized.

Further evidence for a range of densities is that $\mathrm{J}=10 \rightarrow 9$ emission has been seen in a number of sources (Hauschildt et al. 1993 and our Table 4). The data do not warrant detailed source-by-source modeling, but we have predicted the expected $\mathrm{J}=10 \rightarrow 9$ emission from a source with the mean properties found in $\S 4.1$ : $\log (\mathrm{n})=5.93$ and $\log (\mathrm{N})=14.42$. We assumed a linewidth of $5.0 \mathrm{~km} \mathrm{~s}^{-1}$, about the mean for our sample, and $\mathrm{T}_{K}=50 \mathrm{~K}$. The predicted $\mathrm{T}_{R}$ of the $\mathrm{J}=10 \rightarrow 9$ line is $0.2 \mathrm{~K}$ for this average cloud, weaker than any of the detections. If we use the conditions for the cloud with properties at the high end of the $1 \sigma$ spread, we can produce $\mathrm{T}_{R}=1.6 \mathrm{~K}$, about the weakest detection. Increasing $\mathrm{T}_{K}$ to $100 \mathrm{~K}$ raises the prediction to $7 \mathrm{~K}$, similar to many of the detections. Detection of a $\mathrm{J}=10 \rightarrow 9$ line therefore implies a cloud with higher density, column density, and/or temperature than the average cloud in our sample of sources detected at $\mathrm{J}=7 \rightarrow 6$.

\subsection{Masses}

Table 7 contains mass estimates for the regions for which we have determined cloud sizes. We have computed three different estimates. The first estimate assumes that the volume density fills a spherical volume with the diameter of the $\mathrm{J}=5 \rightarrow 4$ emission:

$$
M_{n}=\frac{4}{3} \pi r^{3} n \mu,
$$

where $\mathrm{r}$ is the radius of the cloud and $\mu=2.34 m_{H}$ is the mean mass per particle. The second estimate uses the CS column densities (N) and the formula:

$$
M_{N}=\pi r^{2} \frac{N}{X} \mu
$$

where $\mathrm{X}$ is the abundance of CS. We have used $X=4 \times 10^{-10}$, based on a more detailed analysis of one of the sources in this study (Carr et al. 1995). Finally, we estimated masses from the virial theorem:

$$
M_{V}=\frac{5}{3} \frac{R V_{r m s}^{2}}{G}
$$

for a spherical, non-rotating cloud. Assuming that the velocity profile is Gaussian, $V_{r m s}$ is related to the FWHM $(\Delta v)$ of the line by $V_{r m s}=\sqrt{3} \Delta v / 2.35$. We used the average $\Delta v$ of the CS lines. The value of $M_{n}$ 
for GL490 is probably underestimated substantially because the maser position is quite far from the peak of a very compact source. Zhou et al. (1996) have analyzed this source in more detail and found considerably higher densities from spectra on the peak. Consequently, we ignore this source in the following discussion.

The average ratio of $M_{N} / M_{n}$ is $0.84 \pm 0.73$. The agreement is gratifying, but the poorly known abundance of CS makes $M_{N}$ quite uncertain. In contrast, the agreement between $M_{n}$ and $M_{V}$ is worse, with $M_{n}$ almost always considerably larger than $M_{V}$. A likely explanation is that the gas is distributed inhomogeneously within the beam, whereas the calculation of $M_{n}$ assumes that the density is uniformly distributed. We have used the ratio of $M_{V}$ to $M_{n}$ to estimate the volume filling factor $\left(f_{v}\right)$ of the gas, also listed in Table 7. The filling factors have a large range (0.02 to 2.3) and a mean value of $0.33 \pm 0.59$. The virial mass estimate is susceptible to error because the linewidth may be affected by unbound motions, such as outflows, and it ignores effects of external pressure. Least certain is $M_{n}$, which depends on the cube of the size (and hence distance). Each mass estimate depends on a different power of the size, making their ratio strongly dependent on uncertainties in the distance. In view of the problems inherent in each of the different mass calculations, the masses agree reasonably well. Because the virial mass estimates have the fewest potential problems, we will use them in what follows. The average $M_{V}=3800 \mathrm{M}_{\odot}$.

\section{Implications}

\subsection{Comparison to Other Star-Formation Regions}

Are the high densities seen in this survey peculiar to regions of massive star formation or are they a feature of star formation in general? Lada, Evans, \& Falgarone (1996) have found that the density in the most active star-forming cores in L1630 is about $\log (\mathrm{n})=5.8$, very similar to what we find. We also compared the results of our study with surveys of regions forming low-mass stars. Zhou et al. (1989) observed a sample of low-mass cores in CS transitions up to $\mathrm{J}=5 \rightarrow 4$ and derived densities of $\langle\log (\mathrm{n})\rangle=5.3 \pm 1.1$. These densities are about a factor of 4 lower than the densities we find in this study (and in other studies of regions of massive star formation). Since Zhou et al. (1989) did not have $J=7 \rightarrow 6$ data, it may be more appropriate to compare with our fits to sources without $\mathrm{J}=7 \rightarrow 6$ detections; in that case, our densities are larger by a factor of about 2 . The net result is that regions forming massive stars do seem to have larger densities when similar techniques are used, but the difference is not an order of magnitude.

The ability to form low-mass stars in regions of massive star formation may depend on whether the Jeans mass remains low as the cloud is heated. We can calculate the Jeans mass from

$$
M_{J}\left(\mathrm{M}_{\odot}\right)=18 \mathrm{~T}^{\frac{3}{2}} \mathrm{n}^{-\frac{1}{2}} .
$$

Using the mean logarithmic densities and the assumed temperatures $(10 \mathrm{~K}$ for the low-mass cores, $50 \mathrm{~K}$ for our sample), we compute $\left\langle M_{J}\right\rangle=1.3 \mathrm{M}_{\odot}$ for the clouds forming low-mass stars and $\left\langle M_{J}\right\rangle=7 \mathrm{M}_{\odot}$ for

clouds in this study with $\mathrm{J}=7 \rightarrow 6$ emission. The assumed temperatures make $M_{J}$ higher in regions forming massive stars even though they are denser. However, the strong dependence of $M_{J}$ on temperature means that statements about average properties should not be taken too literally until the temperatures are known better. In addition, the fragmentation spectrum may have been established early in the evolution of the core, before the temperatures were raised by the formation of massive stars. 


\subsection{Do Larson's Laws Apply to Massive Cores?}

Most studies of the global properties of molecular clouds deal with the usual linewidth-size-density relations, as proposed by Larson (1981) and confirmed by others (e.g., Fuller \& Myers 1992; Solomon et al. 1987; Caselli \& Myers 1995). These relations were generally found by comparing properties of whole clouds; similar relations were found within single clouds by comparing map sizes in transitions of different molecules. A recent paper by Caselli \& Myers (1995) includes information on both low mass cores and more massive cores within the Orion molecular cloud. They fit the non-thermal linewidth (the observed linewidth after correction for the thermal contribution) and cloud radius for these types of regions separately to this relation:

$$
\log \Delta v\left(\mathrm{kms}^{-1}\right)=b+q \log R(\mathrm{pc}) .
$$

They found a strong relationship (correlation coefficient, $r=0.81$ ) in low-mass cores with $b=0.18 \pm 0.06$ and $q=0.53 \pm 0.07$. The relation was considerably weaker $(r=0.56)$ and flatter $(q=0.21 \pm 0.03)$ in the massive cores. In Figure 5, we plot $\log (\Delta v)$ versus $\log R$ for the sources in Table 5, which are generally denser and more massive than the cores studied by Caselli \& Myers. No relationship is apparent (the correlation coefficient is only $r=0.26$ ), despite the fact that our sample covers a range of 30 in source size. Nevertheless, we fitted the data to equation 7 using least squares and considering uncertainties in both variables (we assumed $20 \%$ uncertainties in size and used the standard deviation of the linewidths of the different lines for the uncertainty in $\Delta v$ ). The result was $b=0.92 \pm 0.02$ and $q=0.35 \pm 0.03$, but the goodness of fit parameter, $Q=2.8 \times 10^{-8}$, whereas a decent fit should have $Q>0.001$. Alternatively, we minimized the mean absolute deviation (robust estimation; see Press et al. 1992). The result was $b=0.80$ and $q=0.08$, indicating essentially no size-linewidth relation.

Thus our data confirm the trend discernable in the analysis of Caselli \& Myers: the $\Delta v-R$ relation tends to break down in more massive cores. We have plotted the Caselli \& Myers relations in Figure 5, along with Larson's original relation. It is clear that our sources have systematically higher linewidths at a given radius than sources in other studies. For the radii we are probing, most other studies were considering linewidths from $\mathrm{CO}$ or its isotopes and may thus have included a larger contribution from low-density envelopes. The usual relations would predict larger $\Delta v$ in these regions, which would make the discrepancy worse. However, our sources are regions of massive star formation, and Larson (1981) noted that such regions (Orion and M17 in his study) tended to have larger $\Delta v$ for given size and not to show a size-linewidth correlation.

Most previous studies have found an inverse relation between mean density and size, corresponding to a constant column density. However, Scalo (1990) and Kegel (1989) have noted that selection effects and limited dynamic range may have produced this effect, and Leisawitz (1990) found no relationship between density and size in his study of clouds around open clusters. In previous studies, the mean densities were found by dividing a column density by a size, which might be expected to introduce an inverse correlation if the column density tracer has a limited dynamic range. Since our densities were derived from an excitation analysis, it may be interesting to see if any correlation exists in our data. We plot $\log (\mathrm{n})$ versus $\log R$ in Figure 5. Again, no correlation is evident $(r=-0.25)$, and our densities all lie well above (factors of 100!) predictions from previous relations (e.g., Myers 1985). Again, Larson (1981) noted a similar, though

much less dramatic, tendency for regions of massive star formation in his analysis. For a recent theoretical discussion of these relations, see Vázquez-Semadeni, Ballesteros-Paredes, \& Rodríguez (1997).

To use data on sources without size information, we plot (in the bottom panel of Figure 5) $\log (\Delta v)$ versus $\log (\mathrm{n})$. The previous relations would predict a negative slope (typically -0.5$)$ in this relation. In 
contrast to the predictions, our data show a positive, but small, correlation coefficient $(r=0.40)$. The slope from a least squares fit is quite steep $(1.3 \pm 0.2)$, but robust estimation gives a slope of only 0.39 . In addition, the linewidths are much larger than would have been predicted for these densities from previous relations. These results suggests that an uncritical application of scaling relations based on mean densities to actual densities, especially in regions of massive star formation, is likely to lead to errors.

The fact that Larson's laws are not apparent in our data indicate that conditions in these very dense cores with massive star formation are very different from those in more local regions of less massive star formation. The linewidths may have been affected by star formation (outflows, expanding HII regions, etc.); the higher densities are probably caused by gravitational contraction, which will also increase the linewidths. While the regions in this study may not be typical of most molecular gas, they are typical of regions forming most of the massive stars in the Galaxy. These conditions (denser, more turbulent than usually assumed) may be the ones relevant for considerations of initial mass functions.

\subsection{Luminosity, Star Formation Efficiency, and Gas Depletion Time}

We have collected data from the literature (or our own unpublished data) on the luminosity of the sources in Table 7 . The ratio of the luminosity to the virial mass $(L / M)$, roughly proportional to the star formation rate per unit mass, ranges from 24 to 490 in solar units (see Table 7) with a mean of $190 \pm 43$, where 43 is the standard deviation of the mean (all other uncertainties quoted in the text are standard deviations of the distribution). Previous studies, using masses determined from CO luminosity, have found much lower average values of $L / M: 4.0$ for the inner galaxy (Mooney \& Solomon 1988); 1.7 for the outer galaxy (Mead, Kutner, \& Evans 1990). In fact, the maximum values in those samples were 18 and 5, respectively, smaller than any of our values. The enormous difference is caused by the fact that we are calculating the mass of the dense gas, which is much less than the mass computed from the CO luminosity. While we have also tried to use luminosities measured with small beams, the main difference is in the mass. One way to interpret this result is that the star formation rate per unit mass rises dramatically (a factor of $50)$ in the part of the cloud with dense gas.

The star formation rate per unit mass of very dense gas may be more relevant since stars do not seem to form randomly throughout molecular clouds (Lada et al. 1991). Instead, the 4 most massive CS cores in L1630, which cover only $18 \%$ of the surveyed area, contain $58 \%$ to $98 \%$ of all the forming stars, depending on background correction. Li et al. (1996) have found that there is little evidence for any recent star formation outside the clusters, suggesting that the $98 \%$ number is closer to correct. The star formation efficiency in the clusters can be quite high (e.g., 40\%) compared to that of the cloud as a whole (4\%) (Lada et al. 1991).

The gas depletion time $(\tau)$ is the time to turn all the molecular gas into stars. Considering only stars of $M>2 \mathrm{M}_{\odot}$, the star formation rate can be written as $d M / d t\left(\mathrm{M}_{\odot} \mathrm{yr}^{-1}\right)=4 \times 10^{-10} L$ (Gallagher \& Hunter 1986; Hunter et al. 1986). The coefficient differs by only $20 \%$ if the lower mass cutoff is $10 \mathrm{M}_{\odot}$. The gas depletion time can then be written as $\tau=2.5 \times 10^{9}(M / L)$ yr. Using our value of average $L / M=190$, $\tau=1.3 \times 10^{7} \mathrm{yr}$. This time is comparable to that for dispersal of clouds surrounding open clusters; clusters with ages in excess of $1.0 \times 10^{7} \mathrm{yr}$ do not have associated molecular clouds with masses as large as $10^{3} M_{\odot}$ (Leisawitz, Bash, \& Thaddeus 1989). 


\subsection{Luminosity of the Galaxy in CS J $=5 \rightarrow 4$}

CS J $=5 \rightarrow 4$ emission has been seen toward the centers of NGC 253, M82, IC 342, Maffei 2, and NGC 6946 (Mauersberger and Henkel 1989; Mauersberger et al. 1989). For comparison to studies of other galaxies, we will estimate the luminosity of the Milky Way in CS $5 \rightarrow 4\left[L_{G}(\mathrm{CS} 5-4)\right]$ from the mean $L(C S 5-4)$ per cloud in Table 5 and an estimate of the number of such clouds $\left(n_{c l}\right)$ in the Galaxy. From Table 5 we find $\langle L(\mathrm{CS} 5-4)\rangle=4 \times 10^{-2} \mathrm{~L}_{\odot}$ and $\left\langle\int T_{R}^{*} d v\right\rangle=34 \mathrm{~K} \mathrm{~km} \mathrm{~s}^{-1}$, whereas $\left\langle\int T_{R}^{*} d v\right\rangle=42 \mathrm{~K} \mathrm{~km}$ $\mathrm{s}^{-1}$ for the whole sample in Table 2. If we correct for the fact that the mean integrated intensity of the sources in Table 5 is less than the mean of the whole sample, we would get $5 \times 10^{-2} \mathrm{~L} \odot$ for the typical core.

We do not have a direct measurement of $n_{c l}$ because our survey is incomplete. The most recent update to the $\mathrm{H}_{2} \mathrm{O}$ maser catalog (Brand et al. 1994) brings the total number of masers with IRAS colors characteristic of star formation regions (see Palagi et al. 1993) to 414. If we assume that our CS $5 \rightarrow 4$ detection rate of $75 \%$ applies equally to the other sources, we would expect 311 regions of CS $\mathrm{J}=5 \rightarrow 4$ emission in a region which covers two thirds of the galaxy. If we correct for the unsurveyed third of the galaxy, we would estimate the total number of cloud cores emitting CS J $=5 \rightarrow 4$ to be 466 .

Consequently, we will assume $n_{c l}=311-466$, with the larger values probably being more likely. Using these numbers, we calculate $L_{G}(\mathrm{CS} 5-4)=15-23 \mathrm{~L}_{\odot}$. Even though we have made some completeness corrections, we expect these estimates to be underestimates because of our limited sensitivity and the likelihood of $\mathrm{CS}$ emission from dense regions without $\mathrm{H}_{2} \mathrm{O}$ masers.

These values can be compared with the luminosities of other galaxies in Table 8. However, our estimate applies to the entire Galaxy excluding the inner $400 \mathrm{pc}$, while the $L(\mathrm{CS} 5-4)$ for other galaxies are derived from a single beam, centered on the nucleus, with a radius given in the Table. The inner $100 \mathrm{pc}$ of M82 and NGC 253 emit more CS J $=5 \rightarrow 4$ than does our entire Galaxy, excluding the inner 400 pc.

We can also compare our Galaxy to others in terms of its star formation rate per unit mass. In $\S 5.3$, we used $L / M$, with $M$ as the virial mass, to measure this quantity. Because linewidths in galaxy observations are likely to reflect the total mass, rather than the gaseous mass, we will use $L / L(\mathrm{CS} 5-4)$ as a stand-in

for the star formation rate per unit mass of dense gas. We have tabulated the far-infrared luminosity of the galaxies in Table 8, using the data with the smallest available beam, to provide the best match to the CS $\mathrm{J}=5 \rightarrow 4$ observations, which were mostly done with the IRAM $30-\mathrm{m}$ telescope ( $11^{\prime \prime}$ beam). The resulting $L / L(\mathrm{CS} 5-4)$ values range from $5.0 \times 10^{7}(\mathrm{NGC} 253)$ to $1.7 \times 10^{9}(\mathrm{M} 82)$. These numbers apply to regions typically $100 \mathrm{pc}$ in radius. For our Galaxy, we have only the total $L(\mathrm{CS} 5-4)$, so we compare to the total $L=1.8 \times 10^{10} \mathrm{~L} \odot$ (Wright et al. 1991). The result is $8-13 \times 10^{8}$, nominally similar to M82; however, much of the far-infrared emission of our Galaxy is likely to result from heating by older stars. Probably a more useful comparison is to the values of $L / L(\mathrm{CS} 5-4)$ in individual clouds (Table 7 ). No individual cloud approaches the value in M82. The highest value in Table 7 is about twice that of NGC 253 and half that of IC 342 .

\section{Summary}

1. Very dense gas is common in regions of massive star formation. The gas density for the regions selected by having a water maser is $\langle\log (\mathrm{n})\rangle=5.93$ and the CS column density is $\langle\log (\mathrm{N})\rangle=14.42$. 
For regions without CS $\mathrm{J}=7 \rightarrow 6$ emission the mean density is half as large and the mean column density is about 7 times smaller. These results are relatively insensitive to both CS optical depth and to changes in the kinetic temperature of the region. The mean density is an order of magnitude less than the critical density of the $\mathrm{J}=7 \rightarrow 6$ line because of trapping and multilevel excitation effects.

2. In many regions forming massive stars, the CS emission is well modeled by a single density gas component, but many sources also show evidence for a range of densities. From simulations of emission from gas composed of two different densities $\left(10^{4}\right.$ and $\left.10^{8} \mathrm{~cm}^{-3}\right)$, we conclude that there are few clouds with filling factors of ultra-dense gas $\left(\mathrm{n}=10^{8} \mathrm{~cm}^{-3}\right)$ exceeding 0.25 .

3. The densities calculated for the sources in this survey are comparable to the densities seen from detailed studies of a few individual regions forming massive stars. Therefore, it is likely that very dense gas is a general property of such regions. The average density of regions forming massive stars is at least twice the average in regions forming only low-mass stars.

4. Using a subsample of sources whose CS $5 \rightarrow 4$ emission was mapped at the CSO, the average cloud diameter is $1.0 \mathrm{pc}$ and the average virial mass is $3800 \mathrm{M}_{\odot}$.

5. We see no evidence for a correlation between linewidth and size or density and size in our sample. Our linewidths and densities are systematically larger at a given size than those predicted by previous relations. There is, however, a positive correlation between linewidth and density, the opposite of predictions based on the usual arguments.

6. The ratio $L / M$, which is a measure of star formation rate per unit mass for the dense gas probed by CS J $=5 \rightarrow 4$ emission, ranges from 24 to 490, with an average value of 190 .

7. The dense gas depletion time, $\tau \sim 1.3 \times 10^{7} \mathrm{yr}$, comparable to the dispersal time of gas around clusters and OB associations.

8. The estimated Galactic luminosity in the CS $\mathrm{J}=5 \rightarrow 4$ line is $14-23 \mathrm{~L} \odot$. This range of values is considerably less than what is seen in the inner $100 \mathrm{pc}$ of starburst galaxies. In addition, those galaxies have a higher ratio of far-infrared luminosity to CS J $=5 \rightarrow 4$ luminosity than any cloud in our sample.

We are grateful to the staff of the IRAM 30-m telescope for assistance with the observations. We also thank T. Xie, C. M. Walmsley, and J. Scalo for helpful discussion. This research was supported in part by NSF Grant AST-9317567 to the University of Texas at Austin. 
Table 1: Observing Parameters

\begin{tabular}{lccccccc}
\hline \hline Line & $\begin{array}{c}\nu \\
(\mathrm{GHz})\end{array}$ & Telescope & $\eta_{m b}^{a}$ & $\begin{array}{c}\theta_{b}^{a} \\
\left({ }^{\prime \prime}\right)\end{array}$ & $\begin{array}{c}\left\langle T_{\text {sys }}\right\rangle^{b} \\
(\mathrm{~K})\end{array}$ & $\begin{array}{c}\delta v \\
\left(\mathrm{~km} \mathrm{~s}^{-1}\right)\end{array}$ & $\begin{array}{c}\delta v \\
\left(\mathrm{~km} \mathrm{~s}^{-1}\right)\end{array}$ \\
\hline $\mathrm{CS} 2 \rightarrow 1$ & 97.980968 & IRAM & 0.60 & $25^{\prime \prime}$ & 675 & $0.31^{c}$ & $3.06^{d}$ \\
$\mathrm{CS} 3 \rightarrow 2$ & 146.969049 & IRAM & 0.60 & $17^{\prime \prime}$ & 990 & $0.32^{e}$ & $2.04^{d}$ \\
$\mathrm{CS} 5 \rightarrow 4$ & 244.935606 & IRAM & 0.45 & $10^{\prime \prime}$ & 2500 & $1.22^{f}$ & $\ldots$ \\
$\mathrm{C}^{34} \mathrm{~S} 2 \rightarrow 1$ & 96.412982 & IRAM & 0.60 & $25^{\prime \prime}$ & 620 & $0.31^{c, g}$ & $3.11^{d}$ \\
$\mathrm{C}^{34} \mathrm{~S} \mathrm{3 \rightarrow 2}$ & 144.617147 & IRAM & 0.60 & $17^{\prime \prime}$ & 835 & $0.32^{e, h}$ & $2.07^{d}$ \\
$\mathrm{C}^{34} \mathrm{~S} 5 \rightarrow 4$ & 241.016176 & IRAM & 0.45 & $10^{\prime \prime}$ & 2700 & $1.24^{f}$ & $\ldots$ \\
$\mathrm{CS} 5 \rightarrow 4$ & 244.935606 & CSO & 0.71 & $30^{\prime \prime}$ & 445 & $0.17^{i}$ & $1.2^{j}$ \\
$\mathrm{C}^{34} \mathrm{~S} 7 \rightarrow 6$ & 337.396602 & CSO & 0.55 & $20^{\prime \prime}$ & 1000 & $0.12^{i}$ & $0.89^{j}$ \\
$\mathrm{CS} 10 \rightarrow 9$ & 489.75104 & CSO & 0.39 & $14^{\prime \prime}$ & 4300 & $0.09^{i}$ & $0.61^{j}$ \\
$\mathrm{CS} 14 \rightarrow 13$ & 685.434764 & CSO & 0.31 & $11^{\prime \prime}$ & 2050 & $0.06^{i}$ & $0.44^{j}$ \\
& & & & & & &
\end{tabular}

Note. - (a) Efficiency and beam size; (b) average $T_{\text {sys }}$ during observing; (c) $100 \mathrm{kHz}$ filterbank; (d) Split $1 \mathrm{MHz}$ filterbank; (e) Autocorrelator; (f) $1 \mathrm{MHz}$ filterbank; (g) $\Delta V=0.486 \mathrm{~km} \mathrm{~s}^{-1}$ for $\mathrm{C}^{34} \mathrm{~S} 2-1$ in autocorrelator; (h) $\Delta V=0.207 \mathrm{~km} \mathrm{~s}{ }^{-1}$ for $\mathrm{C}^{34} \mathrm{~S} 3-2$ in $100 \mathrm{KHz}$ filterbank; (i) $50 \mathrm{MHz}$ AOS; (j) $500 \mathrm{MHz}$ AOS. 
Table 2: Standin for table 2 ps file. Discard this page.

\begin{tabular}{lrrrrr}
\hline \hline Source & $\int \mathrm{T}_{R^{*}} \mathrm{dV}$ & $V_{L S R}$ & $\mathrm{FWHM}$ & $T_{R}^{*}(10-9)$ & $T_{R}^{*}(14-13)$ \\
& $\mathrm{K} \mathrm{km} \mathrm{s}^{-1}$ & $\mathrm{~km} \mathrm{~s}^{-1}$ & $\mathrm{~km} \mathrm{~s}^{-1}$ & $\mathrm{~K}$ & $\mathrm{~K}$ \\
\hline
\end{tabular}

References. - (a) Carr et al. (1995) 
Table 3: Standin for table 3 ps file. Discard this page.

\begin{tabular}{lrrrrr}
\hline \hline Source & $\int \mathrm{T}_{R^{*}} \mathrm{dV}$ & $V_{L S R}$ & $\mathrm{FWHM}$ & $T_{R}^{*}(10-9)$ & $T_{R}^{*}(14-13)$ \\
& $\mathrm{K} \mathrm{km} \mathrm{s}^{-1}$ & $\mathrm{~km} \mathrm{~s}^{-1}$ & $\mathrm{~km} \mathrm{~s}^{-1}$ & $\mathrm{~K}$ & $\mathrm{~K}$ \\
\hline
\end{tabular}

References. - (a) Carr et al. (1995) 
Table 4: Results for CS $J=10 \rightarrow 9$ and $J=14 \rightarrow 13$ Lines

\begin{tabular}{lccccc}
\hline \hline Source & $\begin{array}{c}\int \mathrm{T}_{R^{*}} \mathrm{dV} \\
\left(\mathrm{K} \mathrm{km} \mathrm{s}^{-1}\right)\end{array}$ & $\begin{array}{c}V_{L S R} \\
\left(\mathrm{~km} \mathrm{~s}^{-1}\right)\end{array}$ & $\begin{array}{c}\mathrm{FWHM} \\
\left(\mathrm{km} \mathrm{s}^{-1}\right)\end{array}$ & $\begin{array}{c}T_{R}^{*}(10-9) \\
(\mathrm{K})\end{array}$ & $\begin{array}{c}T_{R}^{*}(14-13) \\
(\mathrm{K})\end{array}$ \\
\hline GL2591 $^{a}$ & 2.7 & -5.3 & 1.6 & 1.6 & \\
S158A & 22.6 & -57.2 & 2.9 & 7.2 & $\ldots$ \\
W3(2) & 6.4 & -38.49 & 2.28 & 2.6 & $\ldots$ \\
W3(OH) & $\cdots$ & $\cdots$ & $\cdots$ & $\ldots$ & $<1.6$ \\
S255 & 10.3 & 8.2 & 2.3 & 4.4 & $<0.7$
\end{tabular}

References. - (a) Carr et al. (1995) 
Table 5: Diameters, Offsets and Luminosities from CS J $=5 \rightarrow 4$ Maps

\begin{tabular}{|c|c|c|c|c|c|c|c|}
\hline Source & ref & $\begin{array}{l}\text { Dist. } \\
\text { (kpc) }\end{array}$ & $\begin{array}{c}\int \mathrm{T}_{R^{*}} \mathrm{dV} \\
\left(\mathrm{K} \mathrm{km} \mathrm{s} \mathrm{s}^{-1}\right)\end{array}$ & $\begin{array}{c}L(\mathrm{CS} 5-4) \\
\left(10^{-2} \mathrm{~L}_{\odot}\right)\end{array}$ & Beam Corr. & $\begin{array}{l}\text { Diameter } \\
(\mathrm{pc})\end{array}$ & $\begin{array}{c}\text { Offset } \\
(\operatorname{arcsec})\end{array}$ \\
\hline W43S & & 8.5 & 52.8 & 6.1 & 1.5 & 0.9 & $(0,5)$ \\
\hline W43Main1 & & 7.5 & 22.1 & 5.2 & 4.0 & 1.9 & $(20,-36)$ \\
\hline W43Main3 & & 6.8 & 32.4 & 4.6 & 2.9 & 1.4 & $(-8,2)$ \\
\hline $31.25-0.11$ & & 13 & 9.0 & 5.7 & 3.6 & 3.0 & $(-12,-15)$ \\
\hline $31.44-0.26$ & & 9.4 & 23.0 & 8.6 & 4.0 & 2.4 & $(-2,-4)$ \\
\hline $32.8+0.2 \mathrm{~A}$ & & 15 & 64.1 & 15 & 1.0 & $<1.1$ & $(-5,-4)$ \\
\hline W44 & & 3.7 & 87.9 & 3.1 & 2.5 & 0.7 & $(-3,0)$ \\
\hline W51W & & 7 & 12.0 & 1.6 & 2.6 & 1.3 & $(0,-7)$ \\
\hline W51N & & 7 & 79.3 & 17 & 4.2 & 1.8 & $(0,-5)$ \\
\hline W51M & & 7 & 152 & 19 & 2.4 & 1.2 & $(-3,-2)$ \\
\hline ON1 & & 6 & 24.4 & 1.6 & 1.7 & 0.7 & $(0,0)$ \\
\hline K3-50 & & 9 & 11.3 & 1.9 & 2.0 & 1.3 & $(-5,5)$ \\
\hline ON3 & & 9 & 11.0 & 1.8 & 2.0 & 1.3 & $(0,-4)$ \\
\hline $\mathrm{ON} 2 \mathrm{~S}$ & & 5.5 & 22.3 & 1.5 & 2.2 & 0.9 & $(-6,0)$ \\
\hline ON2N & & 5.5 & 15.4 & 1.0 & 2.1 & 0.8 & $(6,5)$ \\
\hline S106 & & 0.6 & 5.4 & 0.004 & 2.2 & 0.1 & $(20,0)$ \\
\hline CRL 2591 & 1 & 1.0 & 7.9 & 0.024 & 3.3 & 0.22 & $(0,0)$ \\
\hline DR21 S & & 3 & 44.8 & 1.0 & 2.3 & 0.5 & $(-6,5)$ \\
\hline $\mathrm{W} 75(\mathrm{OH})$ & & 3 & 47.6 & 1.1 & 2.4 & 0.5 & $(-6,-5)$ \\
\hline W75S1 & & 3 & 9.4 & 0.9 & 9.7 & 1.3 & $(-3,7)$ \\
\hline W75S3 & & 3 & 6.8 & 0.2 & 3.2 & 0.7 & $(0,0)$ \\
\hline W75N & & 3 & 35.2 & 0.8 & 2.5 & 0.5 & $(-5,6)$ \\
\hline CepA & 2 & 0.73 & 30.0 & 0.1 & 5.5 & 0.2 & $(10,12)$ \\
\hline W3(2) & 2 & 2.3 & 26.3 & 0.8 & 5.5 & 0.7 & $(0,12)$ \\
\hline GL 490 & 2 & 0.9 & 7.5 & 0.01 & 1.8 & 0.12 & $(-14,-12)$ \\
\hline
\end{tabular}

References. - (1) Carr et al. (1995); (2) Zhou et al. (1996) 
Table 6: Standin for table 6 ps file. Discard this page.

\begin{tabular}{lrrrrr}
\hline \hline Source & $\int \mathrm{T}_{R^{*}} \mathrm{dV}$ & $V_{L S R}$ & $\mathrm{FWHM}$ & $T_{R}^{*}(10-9)$ & $T_{R}^{*}(14-13)$ \\
& $\mathrm{K} \mathrm{km} \mathrm{s}^{-1}$ & $\mathrm{~km} \mathrm{~s}^{-1}$ & $\mathrm{~km} \mathrm{~s}^{-1}$ & $\mathrm{~K}$ & $\mathrm{~K}$ \\
\hline
\end{tabular}

References. - (a) Carr et al. (1995) 
Table 7: Masses and Luminosities

\begin{tabular}{lccccccccc}
\hline \hline Source & Flag & $\begin{array}{c}M_{n} \\
\left(M_{\odot}\right)\end{array}$ & $\begin{array}{c}M_{N} \\
\left(M_{\odot}\right)\end{array}$ & $\begin{array}{c}M_{V} \\
\left(M_{\odot}\right)\end{array}$ & $f_{v}$ & $\begin{array}{c}L \\
\left(L_{\odot}\right)\end{array}$ & Ref. & $\begin{array}{c}L / M_{V} \\
\left(L_{\odot} / M_{\odot}\right)\end{array}$ & $\begin{array}{c}L / L(\mathrm{CS} 5-4) \\
\left(10^{7}\right)\end{array}$ \\
\hline W43 S & $\mathrm{C}^{34} \mathrm{~S}$ & $2.3 \times 10^{4}$ & $2.8 \times 10^{4}$ & $1.8 \times 10^{3}$ & 0.08 & $\ldots$ & $\ldots$ & $\ldots$ & $\cdots$ \\
$31.44-0.26$ & $\mathrm{C}^{34} \mathrm{~S}$ & $3.9 \times 10^{5}$ & $1.2 \times 10^{5}$ & $6.3 \times 10^{3}$ & 0.02 & $\ldots$ & $\ldots$ & $\ldots$ & $\ldots$ \\
$32.8+0.20 \mathrm{~A}$ & $\mathrm{C}^{34} \mathrm{~S}$ & $5.6 \times 10^{4}$ & $1.3 \times 10^{4}$ & $7.0 \times 10^{3}$ & 0.13 & $\ldots$ & $\ldots$ & $\ldots$ & $\ldots$ \\
W44 & $\mathrm{C}^{34} \mathrm{~S}$ & $1.6 \times 10^{4}$ & $3.9 \times 10^{4}$ & $4.5 \times 10^{3}$ & 0.27 & $3.0 \times 10^{5}$ & 4 & 67 & 1.0 \\
W51 W & $\mathrm{C}^{34} \mathrm{~S}$ & $5.9 \times 10^{4}$ & $1.4 \times 10^{4}$ & $1.5 \times 10^{3}$ & 0.03 & $\ldots$ & $\ldots$ & $\ldots$ & $\ldots$ \\
W51 N C2 & $\mathrm{C}^{34} \mathrm{~S}$ & $6.2 \times 10^{5}$ & $1.2 \times 10^{5}$ & $1.3 \times 10^{4}$ & 0.02 & $4.0 \times 10^{6}$ & 4 & 310 & 2.4 \\
W51M & $\mathrm{CS}$ & $7.2 \times 10^{4}$ & $8.8 \times 10^{4}$ & $1.6 \times 10^{4}$ & 0.23 & $2.8 \times 10^{6}$ & 3 & 170 & 1.5 \\
K3-50 & $\mathrm{C}^{34} \mathrm{~S}$ & $5.9 \times 10^{4}$ & $9.4 \times 10^{3}$ & $6.1 \times 10^{3}$ & 0.10 & $2.1 \times 10^{6}$ & 5 & 340 & 11 \\
ON3 & $\mathrm{C}^{34} \mathrm{~S}$ & $1.7 \times 10^{4}$ & $7.3 \times 10^{3}$ & $2.3 \times 10^{3}$ & 0.13 & $3.7 \times 10^{5}$ & 5 & 160 & 2.1 \\
ON2S & $\mathrm{C}^{34} \mathrm{~S}$ & $3.3 \times 10^{4}$ & $8.0 \times 10^{3}$ & $9.1 \times 10^{2}$ & 0.03 & $\ldots$ & $\ldots$ & $\ldots$ & $\ldots$ \\
$\mathrm{CRL2591}$ & $\mathrm{CS}$ & $3.0 \times 10^{2}$ & $5.0 \times 10^{2}$ & $3.2 \times 10^{2}$ & 1.1 & $2.0 \times 10^{4}$ & 2 & 63 & 8.3 \\
DR21 S & $\mathrm{C}^{34} \mathrm{~S}$ & $3.6 \times 10^{3}$ & $3.5 \times 10^{3}$ & $1.1 \times 10^{3}$ & 0.31 & $5.0 \times 10^{5}$ & 6 & 460 & 5.0 \\
W75(OH) & $\mathrm{C}^{34} \mathrm{~S}$ & $5.6 \times 10^{3}$ & $9.6 \times 10^{3}$ & $1.6 \times 10^{3}$ & 0.27 & $5.4 \times 10^{4}$ & 8 & 35 & 0.5 \\
W75 N & $\mathrm{C}^{34} \mathrm{~S}$ & $6.6 \times 10^{3}$ & $3.8 \times 10^{3}$ & $1.4 \times 10^{3}$ & 0.22 & $1.8 \times 10^{5}$ & 3 & 130 & 2.3 \\
Cep A & $\mathrm{CS}$ & $2.5 \times 10^{2}$ & $4.3 \times 10^{2}$ & $5.9 \times 10^{2}$ & 2.3 & $2.5 \times 10^{4}$ & 1 & 42 & 2.5 \\
W3(2) & $\mathrm{C}^{34} \mathrm{~S}$ & $1.9 \times 10^{4}$ & $2.6 \times 10^{3}$ & $6.1 \times 10^{2}$ & 0.03 & $3.0 \times 10^{5}$ & 7 & 490 & 3.8 \\
GL490 & $\mathrm{CS}$ & 6.2 & $2.8 \times 10^{1}$ & $9.1 \times 10^{1}$ & 15 & $2.2 \times 10^{3}$ & 2 & 24 & 2.2
\end{tabular}

References. - (1) Evans et al. (1981); (2) Mozurkewich et al. (1986); (3) Jaffe, unpublished; (4) Jaffe et al. (1985); (5) Thronson \& Harper (1979); (6) Colomé et al. (1995); (7) Campbell et al. (1995) 
Table 8: Comparison to Other Galaxies

\begin{tabular}{lcccccccc}
\hline \hline Source & $\begin{array}{c}\text { Distance } \\
(\mathrm{Mpc})\end{array}$ & $\begin{array}{c}\text { Radius } \\
(\mathrm{pc})\end{array}$ & $\begin{array}{c}\int T_{R}^{*} d v \\
\left(\mathrm{~K} \mathrm{~km} \mathrm{~s}^{-1}\right)\end{array}$ & $\begin{array}{c}L(\mathrm{CS} 5-4) \\
\left(\mathrm{L}_{\odot}\right)\end{array}$ & Ref. & $\begin{array}{c}L \\
\left(10^{9} \mathrm{~L} \odot\right)\end{array}$ & $\begin{array}{c}\text { Ref. } \\
L / L(\mathrm{CS} 5-4) \\
\left(10^{7}\right)\end{array}$ \\
\hline NGC 253 & $2.5^{a}$ & 67 & 23.5 & 154 & 1 & 8 & 3 & 5 \\
Maffei 2 & 5 & 133 & $<2$ & $<53$ & 2 & 9.5 & 2 & $>18$ \\
IC 342 & $1.8^{b}$ & 48 & 0.76 & 3 & 1 & 0.64 & 4 & 21 \\
M82 & $3.2^{c}$ & 85 & 2.6 & 28 & 1 & 47 & 3 & 170 \\
NGC 6946 & 5 & 133 & $<2.8$ & $<74$ & 2 & 1.2 & 3 & $>1.7$ \\
& & & & & & & & \\
\hline
\end{tabular}

References. - (a) de Vaucouleurs (1978); (b) McCall (1987); (c) Tammann \& Sandage (1968); (1) Mauersberger \& Henkel (1989); (2) Mauersberger et al. (1989); (3) Smith \& Harvey 1996; (4) Becklin et al. (1980) for flux, McCall (1987) for distance. 


\section{REFERENCES}

Becklin, E. E., Gatley, I., Matthews, K., Neugebauer, G., Sellgren, K. Werner, M. W., \& Wynn-Williams, C. G. 1980, ApJ, 236, 441

Benson, P. J., \& Myers, P. C. 1989, ApJS, 71, 89

Bergin, E. A., Snell, R. L., \& Goldsmith, P. F. 1996, ApJ, 460, 343

Brand, J., et al. 1994 A\&AS, 103, 541

Butner, H. M., Evans, N. J., II, Harvey, P. M., Mundy, L. G., Natta, A., \& Randich, M. S. 1990 ApJ, 364, 164

Campbell, M. F., Butner, H. M., Harvey, P. M., Evans, N. J., II, Campbell, M. B., \& Sabbey, C. N. 1995, ApJ, 454, 831

Carr, J. S., Evans, N. J., II, Lacy, J. H., \& Zhou, S. 1995, ApJ, 450, 667

Caselli, P. \& Myers, P. C. 1995, ApJ, 446, 665

Cesaroni, R., Palagi, F., Felli, M., Catarzi, M., Comoretto, G., DiFranco, S., Giovanardi, G., \& Palla, F. 1988, A\&AS, 76, 445

Chin, Y.-N., Henkel, C., Whiteoak, J. B., Langer, N., \& Churchwell, E. B. 1996, A\&A, 305, 960

Churchwell, E. 1990, A. \& A. Review, 2, 79

Churchwell, E., Walmsley, C. M., \& Wood, D. O. S. 1992, A\&A, 253, 541

Colomé, C., Harvey, P. M., Lester, D. F., Campbell, M. F., \& Butner, H. M. 1995, ApJ, 447, 236

Cunningham, C. T., Griffin, M. J., Gee, G., \& Ade, P. A. R. 1984, MNRAS, 210, 891

de Vaucouleurs, G. 1978, ApJ, 224, 79

Elitzur, M., Hollenbach, D. J., \& McKee, C. F. 1989, ApJ, 346, 1983

Elmegreen, B. G. 1985, in Protostars and Planets II, ed. D. C. Black \& M. S. Matthews, (Tucson:University of Arizona), 33

Evans, N. J., II 1989, Rev. Mex. Astr. Ap. 18, 21

Evans, N. J., II, et al. 1981, ApJ, 244, 115

Fuller, G. A., \& Myers, P. C. 1992, ApJ, 384, 523

Gallagher, J. S., \& Hunter, D. A. 1986, in Star Formation in Galaxies, ed. C. J. Lonsdale and S. E. Persson (NASA CP-2466) (Washington: Government Printing Office), 167

Genzel, R., \& Downes, D. 1977, A\&AS, 30, 145

Genzel, R., \& Downes, D. 1979, A\&A, 72, 234

Hauschildt, H., Güsten, R., Phillips, T. G., Schilke, P., Serabyn, E., \& Walker, C. K. 1993, A\&A, 273, L23

Hofner, P., Kurtz, S. Churchwell, E., Walmsley, C. M., \& Cesaroni, R. 1996, ApJ, 460, 359

Hunter, D. A., Gillett, F. C., Gallagher, J. S., III, Rice, W. L., \& Low, F. J. 1986, ApJ, 303, 171

Jaffe, D. T., Genzel, R., Harper, D. A., Harris, A. I., \& Ho, P. T. P. 1985, in IAU Symposium 115, Star Forming Regions, ed. M. Peimbert \& J. Jungaku (Dordrecht: Reidel), 143

Jaffe, D. T., Güsten, R., \& Downes, D. 1981, ApJ, 250, 621 
Jaffe, D. T., Hildebrand R. H., Keene, J., \& Whitcomb, S. E. 1983, ApJ, 273, L89

Jenness, T., Scott, P. F., \& Padman, R. 1995, MNRAS, 276, 1024

Kegel, W. H. 1989, A\&A, 225, 517

Klein, R.I., Sandford, M.T., \& Whitaker, R.W. 1983, ApJ, 271, L69

Kutner, M. L., \& Ulich, B. L. 1981, ApJ, 250, 341

Lada, E. A., DePoy, D. L., Evans, N. J., II, \& Gatley, I. 1991, ApJ, 371, 171

Lada, E. A., Evans, N. J., II, \& Falgarone, E. 1996, submitted to ApJ

LaRosa, T. N. 1983, ApJ, 274, 815

Larson, R. B. 1981, MNRAS, 194, 809

Leisawitz, D. 1990, ApJ, 359, 319

Leisawitz, D., Bash, F. N., \& Thaddeus, P. 1989, ApJS, 70, 731

Li, W., Lada, E. A., \& Evans, N. J., II 1996, in prep.

Makinen, P., Harvey, P. M., Wilking, B. A., \& Evans, N. J., II 1985, ApJ, 299, 341

Mangum, J. G. 1993, PASP, 105, 117

Mauersberger, R., Guélin, M., Martín-Pintado, J., Thum, C., Cernicharo, J., Hein, H., \& Navarro, S. 1989, A\&AS, 79, 217

Mauersberger, R., \& Henkel, C. 1989, A\&A, 223, 79

Mauersberger, R., Henkel, C., Wilson, T. L., \& Harju, J. 1989, A\&A, 226, L5

McCall, M. 1987, AJ, 97, 134

Mead, K. N., Kutner, M. L., \& Evans, N. J., II 1990, ApJ, 354, 492

Mezger, P. G., Chini, R., Kreysa, E., Wink, J. E., \& Salter, C. J. 1988, A\&A,191, 44

Mooney, T. J., \& Solomon, P. M. 1988, ApJ, 334, L51

Mozurkewich, D., Schwartz, P. R. \& Smith, H. A. 1986, ApJ, 311, 377

Mundy, L. G., Evans, N. J., II, Snell, R. L., \& Goldsmith, P. F. 1987, ApJ, 318, 392

Mundy, L. G., Snell, R. L., Evans, N. J., II, Goldsmith, P. F. \& Bally, J. 1986, ApJ, 306, 670

Myers, P. C. 1985, in Protostars and Planets II, ed. D. C. Black \& M. S. Matthews, (Tucson:University of Arizona), 81

Palagi, F., Cesaroni, R., Comoretto, G., Felli, M., \& Natale, V. 1993, A\&AS, 101, 153

Penzias, A. A., \& Burrus, C. A. 1973, ARA\&A, 11, 51

Plume, R., Jaffe, D. T., \& Evans, N. J., II 1992, ApJS, 78, 505

Press, W. H., Teukolsky, S. A., Vetterling, W. T., \& Flannery, B. P. 1992, Numerical Recipes, Second Edition (Cambridge: Cambridge University Press), 694

Richardson, J., White, G. J., Gee, G., Griffin, M. J., Cunningham, C. T., \& Ade P. A. R. 1985, MNRAS, 216,713

Scalo, J. 1990, in Physical Processes in Fragmentation and Star Formation, ed. R. Capuzzo-Dolcetta, C. Chiosi, \& A. di Fazio (Dordrecht:Reidel), 151

Shu, F. H. 1977, ApJ, 214, 488 
Smith, B. J., \& Harvey, P. M. 1996, ApJ, in press (Sept. 1).

Snell, R. L., Mundy, L. G., Goldsmith, P. F., Evans, N. J., II, \& Erickson, N. R. 1984, ApJ, 276,625

Solomon, P. M., Radford, S. J. E., \& Downes, D. 1990, ApJ, 348, L53

Solomon, P. M., Rivolo, A. R., Barrett, J., \& Yahil, A. 1987, ApJ, 319, 730

Strelnitskij, V. S. 1984, MNRAS, 207, 339

Tammann, G. A. \& Sandage, A. 1968, ApJ, 151, 825

Thronson, H. A. \& Harper, D. A. 1979, ApJ, 230, 133

Vázquez-Semadini, E., Ballesteros-Paredes, J., \& Rodríguez, L. F. 1997, ApJ, in press (Jan. 1)

Wang, Y., Jaffe, D. T., Evans, N. J., II, Hayashi, M., Tatematsu, K., \& Zhou, S. 1993, ApJ, 419, 707

Wang, Y., Jaffe, D. T., Graf, U. U., \& Evans, N. J., II 1994, ApJS, 95, 503

Wright, E. L., et al. 1991, ApJ, 381, 200

Wood, D. O. S., \& Churchwell, E. 1989, ApJS, 69, 831.

Zhou, S., Butner, H. M., Evans, N. J., II, Güsten, R., Kutner, M. L., \& Mundy, L. G. 1994, ApJ, 428,219

Zhou, S., Evans, N. J., II, Güsten, R., Mundy, L. G., \& Kutner, M. L. 1991, ApJ, 372, 518

Zhou, S., Evans, N. J., II, Wang, Y., \& Choi, M. 1996, submitted to ApJ

Zhou, S., Wu, Y., Evans, N. J., II, Fuller, G. A., \& Myers, P. C. 1989, ApJ, 346, 168

This preprint was prepared with the AAS IATEX macros v3.0. 
Fig. 1. - (a) Example of a source which we assumed was composed of separate cloud components. Notice that the smaller spectral feature is visible in all transitions except for $\mathrm{C}^{34} \mathrm{~S} 5 \rightarrow 4$ and $7 \rightarrow 6$. (b) Example of a source for which we assumed that the multiple spectral features were a result of self-absorption.

Fig. 2.- Bar graph illustrating the fraction of sources which have $\mathrm{T}_{R}^{*} \geq 0.5 \mathrm{~K}$. Each bar represents a different CS transition. The numbers displayed in each of the bars are the actual number that were detected.

Fig. 3.- The top panel is the distribution of cloud densities as determined from LVG model fits to multiple CS transitions. The solid line shows densities determined from fits to all 4 CS transitions. The dashed line shows densities determined from fits to CS $2 \rightarrow 1,3 \rightarrow 2$, and $5 \rightarrow 4$ only in sources with undetected CS $7 \rightarrow 6$ emission. Bins are 0.1 wide in the log. The bottom panel is the distribution of cloud column densities as determined from LVG model fits to multiple CS transitions. The solid line shows the column densities determined from fits to all 4 CS transitions. The dashed line shows column densities determined from fits to CS $2 \rightarrow 1,3 \rightarrow 2$, and $5 \rightarrow 4$ only in sources with undetected CS $7 \rightarrow 6$ emission. Bins are 0.1 wide in the log.

Fig. 4.- Examples of LVG model fits to the CS line temperatures for 6 sources. The filled circles represent the observations and the solid line plots the line temperatures determined from the LVG model that fitted the observations best. The large error bars on the CS $3 \rightarrow 2$ data points are a result of the large calibration uncertainties for this transition. The dashed lines are the results of fits with two densities, as described in the text.

Fig. 5.- The top plot is the log of the linewidth versus the log of the radius, with the best-fitting straight lines shown as a dash-dotted line (least-squares) and solid line (robust estimation). The other lines are taken from the relations of Larson (1981) and Caselli \& Myers (1995). The middle plot is the log of the density versus the log of the radius. The dashed line is from Myers (1985), assuming that his "size" was a diameter. The bottom plot is the log of the CS linewidths (averaged over all available CS lines) versus the log of the density. Open circles represent the sources with detectable CS $7 \rightarrow 6$ emission and the open pentagons represent those that were not detected in $7 \rightarrow 6$. The filled squares are sources with self absorption. The best-fitting straight line is shown as a solid line (least squares) and as a dotted line (robust estimation). 


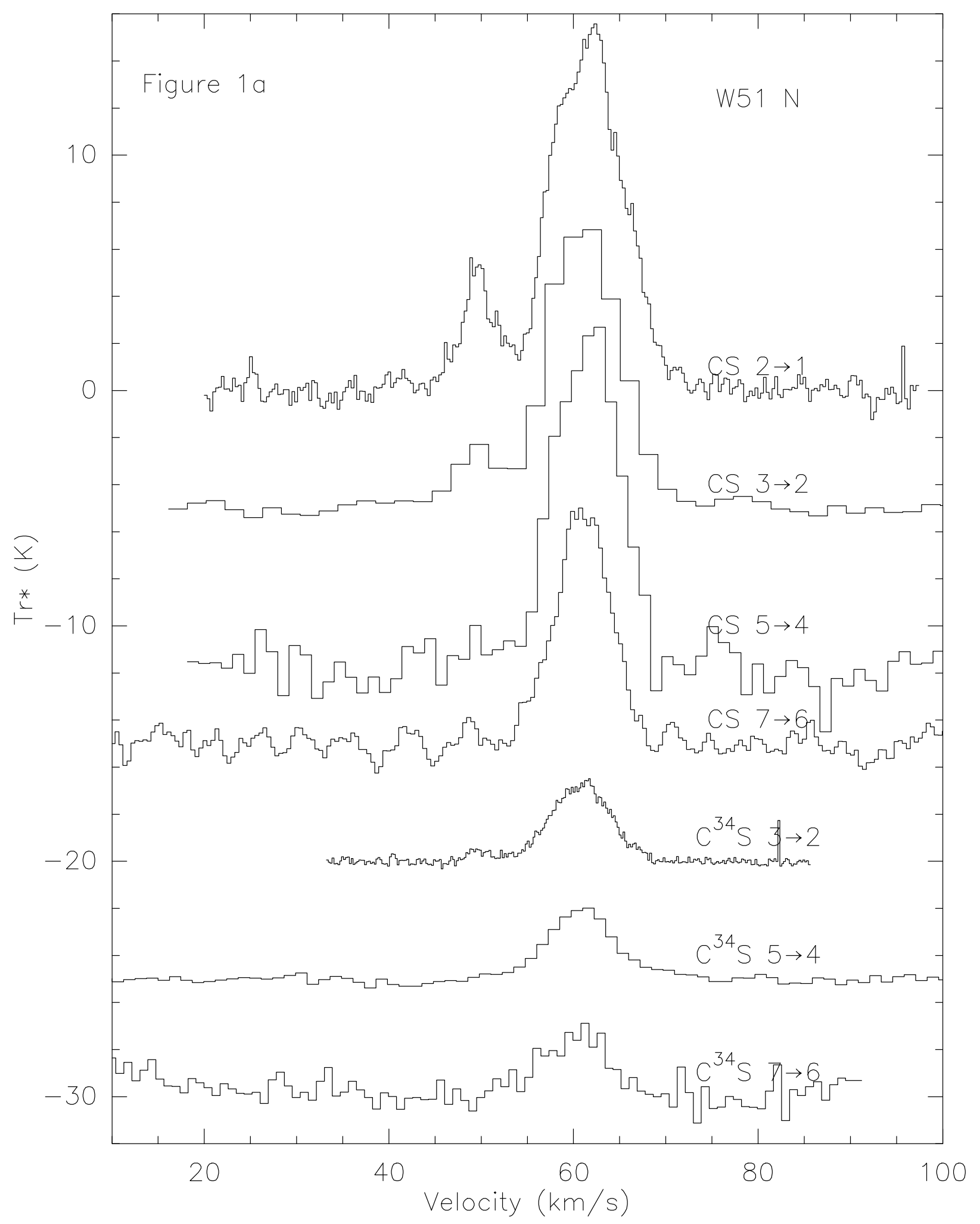




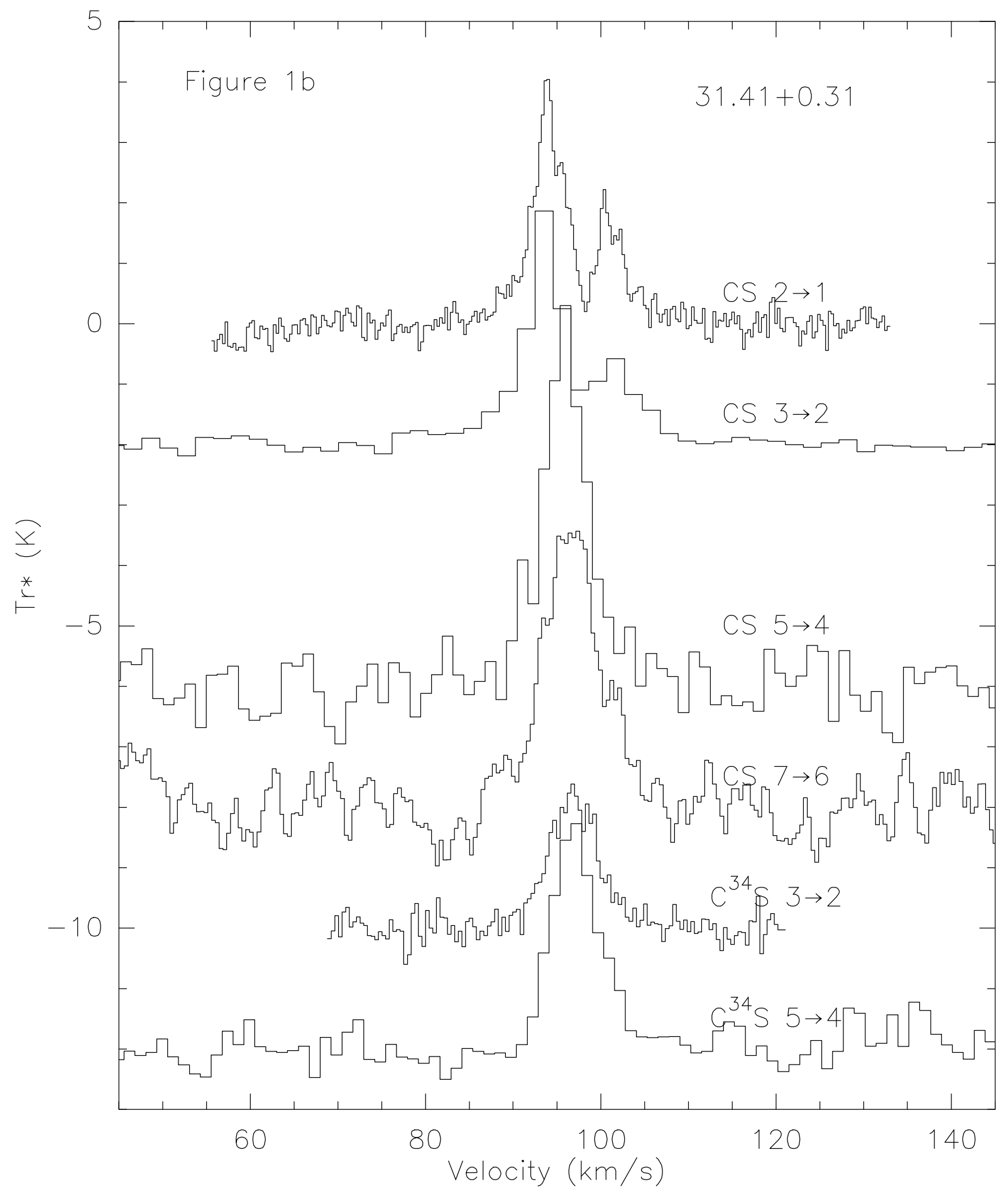


Table 2

CS Source List

\begin{tabular}{|c|c|c|c|c|c|c|c|c|c|c|c|c|c|}
\hline \multirow{3}{*}{$\begin{array}{c}\text { Source } \\
\text { Name }\end{array}$} & \multicolumn{5}{|c|}{ CS J = 2-1 } & \multicolumn{3}{|c|}{ CS J = 3-2 } & \multicolumn{5}{|c|}{ CS J = 5-4 } \\
\hline & Flag & $\mathbf{T}_{\mathbf{R}}^{*}$ & $\int T_{R}^{*} d V$ & VLSR & FWHM & $\mathbf{T}_{\mathbf{R}}^{*}$ & $\int T_{R}^{*} d V$ & VLSR & FWHM & $\mathbf{T R}_{\mathbf{R}}^{*}$ & $\int T_{R}^{*} d V$ & $\mathbf{V}_{\text {LSR }}$ & $\overline{\text { FWHM }}$ \\
\hline & & $\mathbf{K}$ & $\mathrm{K} \mathrm{km} \mathrm{s}^{-1}$ & $\mathbf{k m ~ s}^{-1}$ & $\mathrm{~km} \mathrm{~s}^{-1}$ & $\mathbf{K}$ & $\mathrm{K} \mathrm{km} \mathrm{s}^{-1}$ & $\mathrm{~km} \mathrm{~s}^{-1}$ & $\mathbf{k m ~ s}^{-1}$ & $\mathbf{K}$ & $\mathrm{K} \mathrm{km} \mathrm{s}^{-1}$ & $\mathrm{~km} \mathrm{~s}^{-1}$ & $\mathrm{~km} \mathrm{~s}^{-1}$ \\
\hline RCW 142 & 3 & 22.7 & 158.0 & 16.7 & 6.5 & 22.5 & 172.0 & 16.4 & 7.1 & 18.6 & 182 & 16.1 & 9.2 \\
\hline W28 A2(2) & 3 & 17.9 & 83.8 & 9.3 & 4.4 & 16.5 & 79.9 & 9.3 & 4.6 & 11.8 & 60.4 & 9.3 & 4.8 \\
\hline W28 A2(1) & 2 & 30.8 & 180.0 & 9.6 & 5.5 & 28.1 & 282.0 & 10.5 & 9.4 & 25.5 & 336.0 & 10.9 & 12.4 \\
\hline M8E & 2 & 19.8 & 61.0 & 11.0 & 2.9 & 20.0 & 68.3 & 10.9 & 3.2 & 13.5 & 54.0 & 11.0 & 3.8 \\
\hline $9.62+0.10$ & 2 & $(10.7)$ & 97.1 & 5.2 & 9.1 & (13.2) & 131.7 & 5.4 & 10.0 & (11.1) & 115.8 & 5.6 & 10.4 \\
\hline W31(1) & 3 & (12.6) & 108.3 & 66.5 & 8.6 & (11.5) & 102.0 & 66.3 & 8.9 & (13.3) & 139.5 & 66.0 & 10.5 \\
\hline $10.60-0.40$ & 2 & $(27.2)$ & 236.3 & -2.9 & 8.7 & $(30.4)$ & 301.0 & -3.0 & 9.9 & (27.3) & 286.5 & -2.9 & 10.5 \\
\hline $12.21-0.10$ & 2 & 6.9 & 53.7 & 23.5 & 7.4 & 8.1 & 63.5 & 23.1 & 7.3 & 6.0 & 46.9 & 23.4 & 7.4 \\
\hline $12.42+0.50$ & 2 & 10.7 & 35.1 & 17.2 & 3.1 & 9.7 & 34.1 & 17.0 & 3.4 & 7.1 & 24.7 & 17.1 & 3.3 \\
\hline W33 B & 2 & 3.7 & 11.9 & 57.9 & 3.0 & 3.7 & 8.6 & 57.6 & 2.2 & 3.9 & 21.6 & 54.8 & 5.2 \\
\hline W33 Cont C1 & 2 & 9.0 & 39.4 & 32.7 & 4.1 & 11.5 & 50.2 & 32.9 & 4.1 & 7.6 & 33.2 & 32.9 & 4.1 \\
\hline W33 Cont C2 & 2 & 14.3 & 51.3 & 36.3 & 3.4 & 15.1 & 54.7 & 36.7 & 3.4 & 10.6 & 38.2 & 36.7 & 3.4 \\
\hline W33 Cont C3 & 2 & 8.8 & 29.2 & 40.0 & 3.1 & 10.1 & 33.5 & 40.0 & 3.1 & 7.1 & 23.4 & 40.0 & 3.1 \\
\hline $12.89+0.49$ & 2 & 3.7 & 20.4 & 34.0 & 5.2 & 4.4 & 27.3 & 33.4 & 5.9 & 4.9 & 29.7 & 33.6 & 5.7 \\
\hline $13.87+0.28$ & 2 & 6.4 & 25.9 & 48.6 & 3.8 & 5.1 & 23.1 & 48.4 & 4.1 & 5.2 & 21.0 & 48.6 & 3.8 \\
\hline $14.33-0.64$ & 2 & 5.3 & 42.6 & 22.2 & 7.6 & 8.6 & 67.0 & 22.1 & 7.3 & 10.3 & 81.8 & 22.2 & 7.5 \\
\hline M17(4) & 1 & 25.6 & 118.0 & 19.3 & 4.3 & 17.1 & 92.4 & 19.2 & 5.1 & 19.7 & 114.0 & 19.0 & 5.5 \\
\hline $19.61-0.13$ & 3 & 2.5 & 12.9 & 56.4 & 4.8 & 1.9 & 8.4 & 56.0 & 4.3 & 1.1 & 5.3 & 55.3 & 4.4 \\
\hline $19.61-0.23$ & 3 & 6.3 & 60.5 & 41.7 & 8.9 & 6.3 & 60.9 & 41.7 & 9.1 & 7.3 & 70.6 & 42.2 & 9.1 \\
\hline $20.08-0.13$ & 2 & 4.0 & 29.5 & 40.9 & 6.9 & 4.4 & 41.8 & 40.9 & 8.9 & 3.4 & 30.8 & 40.4 & 8.4 \\
\hline $21.37-0.61$ & 3 & 1.3 & 4.7 & 54.4 & 3.5 & 0.7 & 2.4 & 54.7 & 3.4 & $\leq 0.4$ & - & - & - \\
\hline RCW 169 & 3 & 0.9 & 5.1 & 22.4 & 5.2 & 0.7 & 4.1 & 22.9 & 5.8 & $\leq 0.4$ & - & - & - \\
\hline $22.36+0.07$ & 3 & 3.3 & 11.6 & 84.3 & 3.3 & 2.8 & 9.0 & 84.3 & 3.0 & 2.3 & 7.7 & 84.4 & 3.2 \\
\hline $24.29-0.15$ & 3 & 1.3 & 2.9 & 101.1 & 2.2 & 0.6 & 1.3 & 101.1 & 1.9 & $<0.3$ & - & - & - \\
\hline $24.49-0.04$ & 3 & 6.8 & 41.5 & 109.7 & 5.8 & 5.9 & 39.4 & 109.4 & 6.3 & 3.5 & 24.6 & 109.1 & 6.6 \\
\hline W42 & 2 & $(4.7)$ & 42.4 & 111.1 & 9.0 & $(5.4)$ & 55.7 & 111.4 & 10.4 & $(6.5)$ & 62.9 & 110.4 & 9.7 \\
\hline $28.83-0.25$ & 3 & 1.7 & 7.4 & 87.6 & 4.0 & 1.3 & 5.6 & 87.2 & 4.1 & 1.2 & 5.8 & 87.0 & 4.7 \\
\hline
\end{tabular}


Table 2

CS Source List (Continued)

\begin{tabular}{|c|c|c|c|c|c|c|c|c|c|c|c|c|c|}
\hline \multirow{3}{*}{$\begin{array}{c}\text { Source } \\
\text { Name }\end{array}$} & \multirow{3}{*}{ Flag } & \multicolumn{4}{|c|}{ CS J = 2-1 } & \multicolumn{4}{|c|}{ CS J = 3-2 } & \multicolumn{4}{|c|}{ CS J = 5-4 } \\
\hline & & $\mathbf{T}_{\mathbf{R}}^{*}$ & $\int T_{\mathbf{R}}^{*} \mathrm{dV}$ & VLSR & FWHM & $\mathbf{T}_{\mathbf{R}}^{*}$ & $\int T_{\mathbf{R}}^{*} \mathrm{dV}$ & $\begin{array}{l}\text { LSR } \\
\end{array}$ & FWHM & $\mathbf{T}_{\mathbf{R}}^{*}$ & $\int T_{\mathbf{R}}^{*} \mathrm{dV}$ & $\begin{array}{l}\text { LSR } \\
\end{array}$ & $\overline{\text { FWHM }}$ \\
\hline & & $\mathbf{K}$ & $\mathrm{K} \mathrm{km} \mathrm{s}^{-1}$ & $\mathrm{~km} \mathrm{~s}^{-1}$ & $\mathrm{~km} \mathrm{~s}^{-1}$ & $\mathbf{K}$ & $\mathrm{K} \mathrm{km} \mathrm{s}^{-1}$ & $\mathrm{~km} \mathrm{~s}^{-1}$ & $\mathbf{k m ~ s}^{-1}$ & $\mathbf{K}$ & $\mathrm{K} \mathrm{km} \mathrm{s}^{-1}$ & $\mathrm{~km} \mathrm{~s}^{-1}$ & $\mathbf{k m ~ s}^{-1}$ \\
\hline $28.86+0.07$ & 3 & 6.0 & 28.7 & 103.8 & 4.5 & 5.0 & 27.0 & 103.7 & 5.1 & 3.1 & 14.9 & 103.6 & 4.6 \\
\hline $29.95-0.01$ & 3 & 3.6 & 12.2 & 97.3 & 3.2 & 4.1 & 16.1 & 97.2 & 3.7 & 1.0 & 4.4 & 97.1 & 4.1 \\
\hline W43 S & 1 & 15.6 & 79.8 & 97.5 & 4.8 & 12.2 & 76.7 & 97.6 & 5.9 & 19.0 & 108.0 & 97.9 & 5.3 \\
\hline $30.60-0.04$ & 3 & 0.7 & 0.9 & 89.1 & 1.2 & $<0.4$ & - & - & - & $<0.6$ & - & - & - \\
\hline W43 Main 2 & 3 & $(2.6)$ & 23.6 & 94.7 & 9.1 & $(3.2)$ & 27.5 & 94.7 & 8.5 & $(2.4)$ & 20.3 & 93.4 & 8.6 \\
\hline W43 Main $1 \mathrm{C} 1$ & 1 & 4.9 & 23.9 & 91.4 & 4.6 & 6.0 & 30.4 & 91.4 & 4.8 & 10.2 & 39.7 & 91.8 & 3.6 \\
\hline W43 Main $1 \mathrm{C} 2$ & 1 & 5.8 & 15.3 & 97.4 & 2.5 & 3.4 & 13.9 & 97.4 & 3.9 & 4.9 & 16.5 & 97.2 & 3.2 \\
\hline W43 Main 3 & 1 & $(4.8)$ & 52.0 & 98.6 & 10.8 & $(3.7)$ & 47.6 & 98.9 & 13.0 & $(6.3)$ & 66.8 & 98.4 & 10.6 \\
\hline W43 Main 4 & 2 & 1.2 & 2.7 & 104.4 & 2.1 & 1.0 & 1.9 & 104.4 & 1.8 & $<0.3$ & - & - & - \\
\hline W43(OH) & 3 & $<0.4$ & - & - & - & $<0.3$ & - & - & - & $<0.4$ & - & - & - \\
\hline $31.25-0.11$ & 1 & 4.0 & 19.0 & 21.0 & 4.4 & 2.7 & 13.7 & 20.3 & 4.7 & 1.3 & 13.7 & 21.5 & 9.8 \\
\hline $31.29+0.07$ & 3 & 0.9 & 7.9 & 108.6 & 8.5 & 0.7 & 4.8 & 108.7 & 6.7 & $<0.5$ & - & - & - \\
\hline $31.44-0.26$ & 1 & 10.7 & 53.6 & 87.2 & 4.7 & 6.8 & 39.9 & 87.3 & 5.5 & 5.7 & 41.4 & 87.1 & 6.9 \\
\hline $31.41+0.31$ & 1 & $(2.7)$ & 24.0 & 96.4 & 9.0 & $(2.4)$ & 25.9 & 96.2 & 10.9 & $(5.3)$ & 38.9 & 96.3 & 7.3 \\
\hline $32.05+0.06$ & 3 & $(4.5)$ & 36.2 & 95.0 & 8.1 & $(4.6)$ & 39.4 & 95.1 & 8.5 & $(3.8)$ & 36.0 & 95.7 & 9.4 \\
\hline $32.10-0.08$ & 3 & 1.5 & 4.4 & 47.9 & 2.8 & 0.7 & 2.1 & 48.1 & 2.8 & $\leq 0.4$ & - & - & - \\
\hline $32.74-0.08 \mathrm{~A}$ & 3 & 2.3 & 18.9 & 36.4 & 7.8 & 2.1 & 22.1 & 36.4 & 9.8 & 1.7 & 15.3 & 38.5 & 8.4 \\
\hline $32.8+0.20 \mathrm{~A}$ & 1 & 8.5 & 61.7 & 14.2 & 6.8 & 6.1 & 48.9 & 13.9 & 7.6 & 6.8 & 44.8 & 13.7 & 6.2 \\
\hline $32.80+0.20 \mathrm{~B}$ & 3 & 4.4 & 40.3 & 15.7 & 8.6 & 3.8 & 32.4 & 16.0 & 8.0 & 2.7 & 27.9 & 16.0 & 9.8 \\
\hline $33.81-0.19$ & 2 & 2.9 & 11.8 & 45.9 & 3.8 & 4.0 & 14.6 & 46.0 & 3.4 & 2.7 & 11.7 & 45.5 & 4.1 \\
\hline W44 & 1 & (18.7) & 106.8 & 57.1 & 5.7 & (14.9) & 104.7 & 57.3 & 7.0 & (19.6) & 148.7 & 58.1 & 7.6 \\
\hline $35.20-0.74$ & 2 & $(7.2)$ & 60.5 & 35.1 & 8.4 & $(8.1)$ & 66.3 & 35.1 & 8.2 & $(5.7)$ & 28.1 & 35.8 & 4.9 \\
\hline $35.58-0.03$ & 2 & 6.3 & 33.5 & 53.2 & 5.0 & 6.7 & 49.4 & 52.9 & 6.9 & 7.1 & 40.8 & 53.0 & 5.4 \\
\hline S76 W & 3 & 0.5 & 3.3 & 32.3 & 5.8 & 1.2 & 3.8 & 32.6 & 2.8 & $<0.4$ & - & - & - \\
\hline S76 E & 2 & 15.0 & 49.2 & 33.2 & 3.1 & 17.5 & 70.3 & 32.9 & 3.8 & 12.9 & 54.1 & 32.6 & 4.0 \\
\hline $40.62-0.14$ & 3 & 3.3 & 18.0 & 32.8 & 5.1 & 3.3 & 17.4 & 32.9 & 4.9 & 1.0 & 4.9 & 33.0 & 4.7 \\
\hline W49 S & 2 & 4.9 & 47.9 & 15.1 & 9.1 & 6.9 & 57.5 & 15.3 & 7.8 & 5.7 & 44.4 & 15.5 & 7.3 \\
\hline W49 N C1 & 2 & 9.3 & 89.6 & 3.7 & 9.1 & 10.9 & 111.6 & 3.2 & 9.6 & 13.0 & 152.0 & 5.3 & 11.0 \\
\hline W49 N C2 & 2 & 10.7 & 80.8 & 11.4 & 7.1 & 9.8 & 109 & 11.6 & 10.5 & 6.2 & 59.5 & 14.9 & 9.0 \\
\hline
\end{tabular}


Table 2

CS Source List (Continued)

\begin{tabular}{|c|c|c|c|c|c|c|c|c|c|c|c|c|c|}
\hline \multirow{3}{*}{$\begin{array}{c}\text { Source } \\
\text { Name }\end{array}$} & \multicolumn{5}{|c|}{$\mathrm{CS} \mathrm{J}=2-1$} & \multicolumn{4}{|c|}{$\mathrm{CS} \mathrm{J}=3-2$} & \multicolumn{4}{|c|}{$\mathrm{CS} \mathrm{J}=5-4$} \\
\hline & Flag & $\mathbf{T}_{\mathbf{R}}^{*}$ & $\int T_{R}^{*} d V$ & VLSR & FWHM & $\mathbf{T}_{\mathbf{R}}^{*}$ & $\int T_{R}^{*} d V$ & VLSR & FWHM & $\mathbf{T}_{\mathbf{R}}^{*}$ & $\int T_{\mathbf{R}}{ }^{*} \mathrm{dV}$ & VLSR $_{\text {LS }}$ & $\overline{\text { FWHM }}$ \\
\hline & & $\mathbf{K}$ & $\mathrm{K} \mathrm{km} \mathrm{s}^{-1}$ & $\mathrm{~km} \mathrm{~s}^{-1}$ & $\mathrm{~km} \mathrm{~s}^{-1}$ & $\mathbf{K}$ & $\mathrm{K} \mathrm{km} \mathrm{s}^{-1}$ & $\mathrm{~km} \mathrm{~s}^{-1}$ & $\mathbf{k m ~ s}^{-1}$ & $\mathbf{K}$ & $\mathrm{K} \mathrm{km} \mathrm{s}^{-1}$ & $\mathrm{~km} \mathrm{~s}^{-1}$ & $\mathrm{~km} \mathrm{~s}^{-1}$ \\
\hline $\mathrm{OH} 43.8-0.10$ & 2 & 7.3 & 50.2 & 44.3 & 6.5 & 8.6 & 63.8 & 44.5 & 7.0 & 5.9 & 45.8 & 44.6 & 7.3 \\
\hline $45.07+0.13$ & 2 & 6.3 & 36.9 & 58.9 & 5.5 & 8.1 & 50.5 & 58.9 & 5.9 & 5.9 & 35.0 & 58.8 & 5.6 \\
\hline $48.61+0.02$ & 2 & 4.0 & 25.6 & 17.8 & 6.0 & 4.9 & 28.9 & 17.9 & 5.5 & 3.0 & 20.2 & 18.1 & 6.3 \\
\hline W51 W & 1 & 8.0 & 35.8 & 51.0 & 4.2 & 5.7 & 28.9 & 50.6 & 4.7 & 7.8 & 28.6 & 50.7 & 3.4 \\
\hline W51 N C1 & 1 & 4.9 & 20.3 & 49.5 & 3.9 & 2.7 & 11.4 & 50.0 & 4.0 & 1.4 & 5.9 & 50.0 & 4.0 \\
\hline W51 N C2 & 1 & 15.0 & 144.0 & 61.5 & 9.0 & 12.3 & 116.0 & 61.1 & 8.9 & 14.8 & 129.0 & 61.5 & 8.2 \\
\hline W51 M & 1 & 23.8 & 259.0 & 55.7 & 10.2 & 18.8 & 220.0 & 55.5 & 11.0 & 24.7 & 309.0 & 56.2 & 11.7 \\
\hline $59.78+0.06$ & 2 & 8.3 & 22.5 & 22.6 & 2.5 & 7.9 & 23.0 & 22.6 & 2.7 & 3.5 & 9.4 & 22.7 & 2.5 \\
\hline S87 & 2 & 11.1 & 45.8 & 21.8 & 3.9 & 10.7 & 46.5 & 22.3 & 4.1 & 7.9 & 35.7 & 21.9 & 4.3 \\
\hline S88 B & 3 & 3.8 & 10.9 & 21.8 & 2.7 & 6.3 & 17.9 & 21.8 & 2.7 & 2.4 & 8.4 & 22.0 & 3.2 \\
\hline ON 1 & 1 & (5.8) & 34.5 & 11.5 & 5.9 & (3.9) & 26.1 & 11.5 & 6.7 & $(6.8)$ & 42.6 & 11.7 & 6.3 \\
\hline K3-50 & 1 & 4.7 & 41.8 & -43.4 & 8.4 & 4.2 & 35.0 & -43.8 & 7.9 & 4.3 & 30.0 & -44.1 & 6.6 \\
\hline ON 3 & 1 & (4.0) & 24.3 & -22.5 & 6.1 & $(2.4)$ & 13.6 & -22.1 & 5.7 & $(2.5)$ & 13.4 & -21.7 & 5.4 \\
\hline $\mathrm{ON} 2 \mathrm{~S}$ & 1 & 12.8 & 76.4 & -1.3 & 5.6 & 10.1 & 65.9 & -1.5 & 6.1 & 10.0 & 61.9 & -1.6 & 5.8 \\
\hline ON 2N & 1 & 10.5 & 55.9 & 0.3 & 5.0 & 6.1 & 44.2 & 0.2 & 6.9 & 7.5 & 47.0 & 0.6 & 5.9 \\
\hline S106 & 1 & 3.4 & 17.1 & -1.5 & 4.8 & 2.6 & 14.7 & -2.0 & 5.3 & 3.0 & 24.2 & -2.8 & 7.5 \\
\hline CRL 2591 & 2 & 11.2 & 40.3 & -5.9 & 3.6 & 13.6 & 50.4 & -5.7 & 3.4 & 11.1 & 40.8 & -5.7 & 3.5 \\
\hline DR21 S & 1 & 12.5 & 53.2 & -2.6 & 4.0 & 7.2 & 53.4 & -2.6 & 7.0 & 8.3 & 76.1 & -2.4 & 8.7 \\
\hline $\mathrm{W} 75(\mathrm{OH})$ & 1 & $(18.0)$ & 108.1 & -3.2 & 6.0 & (12.5) & 84.8 & -3.3 & 6.8 & (20.2) & 137.3 & -3.0 & 6.8 \\
\hline W75 S1 & 1 & 9.5 & 43.5 & -4.0 & 4.3 & 4.8 & 28.0 & -3.6 & 5.5 & 7.2 & 27.3 & -3.1 & 3.6 \\
\hline W75 S3 & 1 & 6.9 & 32.4 & -4.3 & 4.4 & 3.6 & 22.7 & -4.1 & 5.9 & 4.0 & 20.3 & -4.1 & 4.8 \\
\hline W75 N & 1 & 15.8 & 85.8 & 9.4 & 5.1 & 11.1 & 69.4 & 9.5 & 5.9 & 17.3 & 96.5 & 9.6 & 5.3 \\
\hline GL 2789 & 1 & 4.8 & 13.4 & -43.8 & 2.6 & - & - & - & - & 2.5 & 7.5 & -43.8 & 2.8 \\
\hline S128 & 1 & 1.5 & 4.3 & -73.6 & 2.8 & 0.6 & 2.2 & -73.4 & 3.2 & 0.7 & 1.6 & -73.6 & 2.0 \\
\hline $97.34+3.25$ & 2 & 0.9 & 6.2 & -72.3 & 6.7 & 1.0 & 5.8 & -73.2 & 5.3 & $\leq 0.4$ & - & -73 & - \\
\hline $97.53+3.17$ & 1 & 3.2 & 10.8 & -71.9 & 3.1 & - & - & - & - & $<4.0$ & - & - & - \\
\hline $97.53+3.19$ & 1 & $(4.5)$ & 23.6 & -70.2 & 5.3 & (3.3) & 17.7 & -69.8 & 5.3 & $(3.0)$ & 15.4 & -69.2 & 5.1 \\
\hline IRAS $21519+5613$ & 2 & 2.7 & 7.9 & -63.0 & 2.8 & 3.7 & 10.9 & -63.0 & 2.8 & 2.2 & 8.2 & -63.1 & 3.5 \\
\hline IRAS $21512+5625$ & 3 & 0.8 & 1.8 & -61.7 & 2.1 & 1.1 & 2.6 & -61.7 & 2.2 & 0.8 & 2.4 & -62.1 & 2.9 \\
\hline
\end{tabular}


Table 2

CS Source List (Continued)

\begin{tabular}{|c|c|c|c|c|c|c|c|c|c|c|c|c|c|}
\hline & Flag & & $\mathbf{C S ~ J ~ = ~}$ & -1 & & & $\mathbf{C S ~ J ~ = ~}$ & $3-2$ & & & $\mathbf{C S ~ J ~ = ~}$ & -4 & \\
\hline Source & & $\mathbf{T}_{\mathbf{R}}^{*}$ & $\int T_{R}^{*} d V$ & VLSR & FWHM & $\mathbf{T} \mathbf{R}^{*}$ & $\int T_{R}^{*} d V$ & VLSR $_{\text {LS }}$ & FWHM & $\mathbf{T} \mathbf{R}^{*}$ & $\int T_{R}^{*} d V$ & VLSR & $\overline{\text { FWHM }}$ \\
\hline Name & & $\mathbf{K}$ & $\mathrm{K} \mathrm{km} \mathrm{s}^{-1}$ & $\mathbf{k m ~ s}^{-\mathbf{1}}$ & $\mathbf{k m ~ s}^{-\mathbf{1}}$ & $\mathbf{K}$ & $\mathrm{K} \mathrm{km} \mathrm{s}^{-1}$ & $\mathbf{k m ~ s}^{-1}$ & $\mathbf{k m ~ s}^{-1}$ & $\mathbf{K}$ & $\mathrm{K} \mathrm{km} \mathrm{s}^{-1}$ & $\mathrm{~km} \mathrm{~s}^{-1}$ & $\mathbf{k m ~ s}^{-1}$ \\
\hline IRAS $21542+5558$ & 3 & 1.9 & 1.9 & -48.5 & 0.9 & 1.0 & 1.3 & -48.4 & 1.3 & $<0.4$ & - & - & - \\
\hline IRAS $22142+5206$ & 3 & 1.6 & 3.4 & -37.4 & 2.1 & 1.6 & 3.3 & -37.3 & 1.9 & 0.6 & 1.7 & -37.2 & 2.9 \\
\hline BFS 10 & 3 & 2.5 & 7.2 & -63.9 & 2.7 & 2.7 & 7.6 & -64.1 & 2.6 & 1.7 & 4.1 & -63.9 & 2.3 \\
\hline IRAS $21561+5806$ & 3 & 2.2 & 6.9 & -82.3 & 2.9 & 2.0 & 5.9 & -82.4 & 2.8 & 0.6 & 1.5 & -83.2 & 2.2 \\
\hline IRAS 21558+5907 & 3 & 1.7 & 3.5 & -88.8 & 1.9 & 1.6 & 4.9 & -88.6 & 2.8 & 0.9 & 2.2 & -89.2 & 2.2 \\
\hline IRAS $22172+5549$ & 3 & 3.2 & 10.3 & -43.1 & 3.0 & 3.7 & 10.2 & -43.3 & 2.6 & 1.1 & 3.2 & -43.5 & 2.7 \\
\hline IRAS $22181+5716$ & 3 & 0.9 & 2.1 & -59.0 & 2.2 & 0.7 & 1.9 & -58.8 & 2.6 & $<0.2$ & - & - & - \\
\hline IRAS 22189+5719 & 3 & 1.2 & 2.9 & -63.5 & 2.2 & 0.9 & 2.6 & -63.3 & 2.6 & $<0.4$ & - & - & - \\
\hline IRAS 22134+5834 & 3 & 3.4 & 9.3 & -18.3 & 2.6 & 3.8 & 9.5 & -18.2 & 2.4 & 2.0 & 3.6 & -18.1 & 1.7 \\
\hline BFS 11-A & 1 & 1.8 & 2.1 & -10.4 & 1.1 & 1.0 & 2.8 & -10.0 & 2.6 & $<1.0$ & - & - & - \\
\hline BFS 11-B & 1 & 3.7 & 11.6 & -10.0 & 2.9 & 3.1 & 12.8 & -9.9 & 3.8 & 4.5 & 19.8 & -9.4 & 4.2 \\
\hline IRAS $22305+5803$ & 2 & 2.0 & 4.3 & -51.9 & 2.4 & 2.5 & 7.7 & -51.8 & 2.9 & 2.5 & 5.1 & -52.0 & 1.6 \\
\hline IRAS $22308+5812$ & 2 & 4.3 & 15.2 & -51.9 & 3.4 & 2.7 & 14.1 & -52.1 & 4.9 & 1.2 & 7.2 & -51.6 & 5.4 \\
\hline IRAS $22365+5818$ & 2 & $<0.5$ & - & - & - & $<0.3$ & - & - & - & $<0.5$ & - & - & - \\
\hline IRAS 23004+5642 & 3 & 1.2 & 1.7 & -53.5 & 1.3 & 0.7 & 0.9 & -53.5 & 1.2 & $<0.5$ & - & - & - \\
\hline Cep A & 2 & $(8.0)$ & 37.6 & -10.5 & 4.7 & $(10.0)$ & 53.8 & -10.6 & 5.4 & $(11.1)$ & 59.8 & -10.5 & 5.4 \\
\hline S157 & 1 & 5.6 & 16.2 & -43.9 & 2.7 & 6.5 & 29.2 & -44.5 & 4.2 & 5.6 & 19.9 & -44.4 & 3.3 \\
\hline S158 & 1 & 15.1 & 85.4 & -56.9 & 5.3 & 11.3 & 72.1 & -57.0 & 5.9 & 13.9 & 63.4 & -57.2 & 4.3 \\
\hline $\mathrm{S} 158 \mathrm{~A}$ & 1 & 15.9 & 67.7 & -57.1 & 4.0 & 11.5 & 63.0 & -57.0 & 5.1 & 16.9 & 77.5 & -56.9 & 4.3 \\
\hline IRAS 23314+6033 & 3 & 1.9 & 4.0 & -45.6 & 2.0 & 1.9 & 4.6 & -45.7 & 2.3 & $\leq 0.7$ & - & - & - \\
\hline IRAS 23385+6053 & 2 & 3.3 & 10.4 & -49.4 & 3.0 & 3.2 & 10.7 & -49.4 & 3.2 & $\leq 1.4$ & - & - & - \\
\hline $118.96+1.88$ & 3 & 3.4 & 9.9 & -36.0 & 2.7 & 3.0 & 8.4 & -35.9 & 2.6 & 1.3 & 3.2 & -36.1 & 2.3 \\
\hline IRAS 00117+6412 & 3 & 3.1 & 9.4 & -36.2 & 2.8 & 3.3 & 8.1 & -36.1 & 2.3 & 1.5 & 5.1 & -36.1 & 3.2 \\
\hline IRAS $00211+6549$ & 3 & 2.9 & 14.2 & -68.9 & 4.6 & 4.3 & 11.7 & -68.8 & 2.6 & 1.5 & 5.1 & -68.6 & 3.1 \\
\hline IRAS 00338+6312 & 1 & $(8.4)$ & 37.9 & -17.9 & 4.5 & $(6.2)$ & 31.4 & -17.7 & 5.1 & $(4.7)$ & 23.8 & -17.4 & 5.1 \\
\hline IRAS 00342+6347 & 3 & 1.6 & 5.2 & -28.0 & 3.0 & 1.0 & 3.2 & -28.5 & 3.0 & $<0.3$ & - & - & - \\
\hline $121.38+1.23$ & 3 & 0.8 & 3.8 & -27.1 & 4.5 & 0.7 & 0.5 & -28.4 & 0.7 & $<0.3$ & - & - & - \\
\hline IRAS 00379+6248 & 3 & 5.5 & 8.7 & -17.8 & 1.5 & 5.4 & 8.6 & -17.9 & 1.5 & 2.5 & 3.9 & -17.6 & 1.5 \\
\hline IRAS 00468+6527 & 3 & 1.5 & 4.9 & -63.4 & 3.0 & 1.8 & 4.2 & -63.2 & 2.2 & 1.3 & 3.0 & -63.6 & 2.2 \\
\hline
\end{tabular}


Table 2

CS Source List (Continued)

\begin{tabular}{|c|c|c|c|c|c|c|c|c|c|c|c|c|c|}
\hline \multirow{3}{*}{$\begin{array}{c}\text { Source } \\
\text { Name }\end{array}$} & \multicolumn{2}{|l|}{ Flag } & \multicolumn{2}{|c|}{ CS J = 2-1 } & \multirow{3}{*}{$\begin{array}{c}\text { FWHM } \\
\mathrm{km} \mathrm{s}^{-1} \\
\end{array}$} & \multicolumn{4}{|c|}{$\mathrm{CS} \mathrm{J}=3-2$} & \multicolumn{4}{|c|}{ CS J = 5-4 } \\
\hline & & $\mathbf{T}_{\mathbf{R}}^{*}$ & $\int T_{R}^{*} d V$ & VLSR & & $\mathbf{T}_{\mathbf{R}}^{*}$ & $\int T_{R}{ }^{*} d V$ & VLSR & FWHM & $\mathbf{T}_{\mathbf{R}}^{*}$ & $\int T_{R}{ }^{*} d V$ & VLSR & $\overline{\text { FWHM }}$ \\
\hline & & $\mathbf{K}$ & $\mathrm{K} \mathrm{km} \mathrm{s}^{-1}$ & $\mathrm{~km} \mathrm{~s}^{-1}$ & & $\mathbf{K}$ & $\mathrm{K} \mathrm{km} \mathrm{s}^{-1}$ & $\mathrm{~km} \mathrm{~s}^{-1}$ & $\mathrm{~km} \mathrm{~s}^{-1}$ & $\mathbf{K}$ & $\mathrm{K} \mathrm{km} \mathrm{s}^{-1}$ & $\mathrm{~km} \mathrm{~s}^{-1}$ & $\mathrm{~km} \mathrm{~s}^{-1}$ \\
\hline NGC 281 & 1 & 9.1 & 38.8 & -30.9 & 4.0 & 6.4 & 36.4 & -31.5 & 5.4 & 5.9 & 31.9 & -31.1 & 5.1 \\
\hline IRAS $01045+6505$ & 3 & 1.6 & 5.1 & -86.6 & 3.1 & 1.8 & 7.1 & -86.3 & 3.6 & 1.6 & 4.3 & -86.0 & 2.6 \\
\hline IRAS $01123+6430$ & 3 & 2.2 & 5.6 & -54.7 & 2.4 & 1.9 & 5.0 & -54.8 & 2.5 & 0.8 & 2.4 & -55.1 & 2.8 \\
\hline IRAS $01134+6429$ & 3 & 1.5 & 2.1 & -54.4 & 1.3 & 1.8 & 2.8 & -54.5 & 1.4 & $\leq 0.9$ & - & - & - \\
\hline $\mathrm{W} 3(1)$ & 1 & 7.7 & 27.4 & -43.0 & 3.3 & 5.0 & 22.7 & -43.3 & 4.3 & 3.8 & 15.1 & -43.5 & 3.7 \\
\hline W3(2) & 1 & (5.1) & 28.3 & -38.6 & 5.5 & $(5.3)$ & 31.1 & -38.7 & 5.9 & $(9.3)$ & 50.2 & -38.5 & 5.4 \\
\hline W3(3) & 1 & 5.8 & 23.4 & -39.0 & 3.8 & 4.0 & 22.8 & -39.2 & 5.3 & 5.0 & 25.5 & -38.7 & 4.8 \\
\hline $\mathrm{W} 3(\mathrm{OH})$ & 1 & (12.6) & 73.1 & -47.3 & 5.8 & $(10.5)$ & 73.8 & -47.6 & 7.0 & $(16.4)$ & 113.8 & -47.4 & 6.9 \\
\hline IRAS $02310+6133$ & 3 & 2.5 & 5.2 & -40.0 & 2.0 & 2.1 & 5.4 & -40.0 & 2.4 & $<0.5$ & - & - & - \\
\hline IRAS $02395+6944$ & 2 & 3.0 & 9.4 & -71.2 & 3.0 & 3.0 & 8.6 & -71.2 & 2.7 & 2.2 & 6.5 & -71.4 & 2.8 \\
\hline IRAS $02333+5930$ & 3 & 1.5 & 2.4 & -26.5 & 1.5 & 1.5 & 3.0 & -26.3 & 1.9 & $\leq 0.5$ & - & - & - \\
\hline IRAS $02461+6147$ & 1 & 3.0 & 9.6 & -42.4 & 3.0 & 2.3 & 9.1 & -42.6 & 3.8 & 2.5 & 7.3 & -42.4 & 2.7 \\
\hline IRAS $02541+6208$ & 1 & 2.5 & 8.4 & -52.1 & 3.2 & 2.0 & 8.2 & -52.3 & 3.9 & 2.7 & 13.1 & -52.4 & 4.5 \\
\hline S201 & 1 & 4.2 & 8.8 & -37.9 & 2.0 & 2.0 & 7.4 & -38.2 & 3.5 & 1.9 & 5.5 & -37.9 & 2.7 \\
\hline $140.64+0.67$ & 3 & 4.1 & 7.9 & -38.0 & 1.8 & 3.5 & 6.6 & -38.1 & 1.8 & 1.7 & 1.6 & -38.0 & 0.9 \\
\hline IRAS $03101+5821$ & 1 & 5.2 & 10.4 & -38.2 & 1.9 & 1.9 & 5.7 & -38.5 & 2.8 & $\leq 1.2$ & - & - & - \\
\hline GL 490 & 3 & 4.1 & 11.3 & -13.2 & 2.6 & 3.3 & 9.9 & -13.2 & 2.8 & 0.8 & 2.2 & -13.1 & 2.6 \\
\hline IRAS $03353+5550$ & 3 & 0.7 & 0.6 & -41.7 & 0.8 & 0.7 & 0.7 & -41.7 & 0.9 & $<0.3$ & - & - & - \\
\hline $145.39+4.01$ & 1 & $<0.5$ & - & - & - & $<0.6$ & - & - & - & $<1.0$ & - & - & - \\
\hline IRAS $03534+5402$ & 3 & 0.6 & 0.4 & -50.7 & 0.7 & 0.5 & 1.0 & -51.0 & 1.9 & $<0.2$ & - & - & - \\
\hline IRAS $04070+5411$ & 3 & 1.0 & 1.3 & -41.4 & 1.3 & 1.4 & 1.8 & -41.3 & 1.2 & $\leq 0.3$ & - & - & - \\
\hline IRAS $04146+5318$ & 3 & 1.0 & 1.6 & -41.5 & 1.5 & 1.0 & 0.8 & -41.8 & 0.7 & $<0.5$ & - & - & - \\
\hline S209 & 3 & 1.5 & 3.7 & -49.4 & 2.4 & 1.4 & 3.0 & -49.3 & 2.0 & 1.4 & 1.5 & -49.0 & 1.0 \\
\hline IRAS $03245+3002$ & 1 & 5.7 & 9.3 & 4.9 & 1.5 & 2.4 & 6.7 & 4.7 & 2.6 & $<1.5$ & - & - & - \\
\hline $\mathrm{S} 231$ & 3 & 8.3 & 35.4 & -16.3 & 4.0 & 9.0 & 38.3 & -15.9 & 4.0 & 5.4 & 26.5 & -15.9 & 4.6 \\
\hline $\mathrm{S} 235$ & 1 & 16.3 & 49.2 & -17.1 & 2.8 & 13.3 & 42.2 & -17.0 & 3.0 & 9.2 & 36.7 & -16.9 & 3.8 \\
\hline S241 & 1 & 6.5 & 16.6 & -8.8 & 2.4 & 5.0 & 14.7 & -8.8 & 2.8 & 4.0 & 10.4 & -8.5 & 2.3 \\
\hline $\mathrm{S} 252 \mathrm{~A}$ & 1 & 10.0 & 34.5 & 9.2 & 3.2 & 7.5 & 31.7 & 9.2 & 4.0 & 6.1 & 25.3 & 9.5 & 3.9 \\
\hline $\mathrm{S} 255 / 7$ & 1 & 19.6 & 64.3 & 7.3 & 3.1 & 14.1 & 60.2 & 7.4 & 4.0 & 12.0 & 47.8 & 7.7 & 3.7 \\
\hline
\end{tabular}


Table 2

CS Source List (Continued)

\begin{tabular}{|c|c|c|c|c|c|c|c|c|c|c|c|c|c|}
\hline \multirow{3}{*}{$\begin{array}{c}\text { Source } \\
\text { Name }\end{array}$} & \multicolumn{5}{|c|}{$\overline{C S J}=2-1$} & \multicolumn{4}{|c|}{$\overline{C S S ~ J ~=~ 3-2 ~}$} & \multicolumn{4}{|c|}{$\overline{C S S ~ J ~=~ 5-4 ~}$} \\
\hline & Flag & $\mathbf{T} \mathbf{R}^{*}$ & $\int T_{R}^{*} d V$ & VLSR $_{\text {LSR }}$ & FWHM & $\mathbf{T R}^{*}$ & $\int T_{R} * d V$ & VLSR & FWHM & $\mathbf{T} \mathbf{R}^{*}$ & $\int T_{R}^{*} d V$ & VISR & FWHM \\
\hline & & $\mathbf{K}$ & $\mathrm{K} \mathrm{km} \mathrm{s}^{-1}$ & $\mathrm{~km} \mathrm{~s}^{-\mathbf{1}}$ & $\mathrm{km} \mathrm{s}^{-\mathbf{1}}$ & $\mathbf{K}$ & $\mathrm{K} \mathrm{km} \mathrm{s}^{-1}$ & $\mathbf{k m ~ s}^{-1}$ & $\mathbf{k m ~ s} \mathbf{s}^{-1}$ & $\mathbf{K}$ & $\mathrm{K} \mathrm{km} \mathrm{s}^{-1}$ & $\mathrm{~km} \mathrm{~s}^{-1}$ & $\mathrm{~km} \mathrm{~s}^{-1}$ \\
\hline $195.82-0.21$ & 3 & 1.4 & 2.5 & 30.2 & 1.7 & 1.3 & 2.0 & 30.1 & 1.4 & $<0.7$ & - & - & - \\
\hline S266 & 3 & 0.9 & 2.5 & 34.1 & 2.7 & 1.4 & 3.3 & 33.8 & 2.2 & $<0.4$ & - & - & - \\
\hline S269 B & 1 & 2.6 & 6.9 & 12.1 & 2.5 & 1.4 & 6.7 & 12.1 & 4.5 & 1.3 & 3.7 & 11.6 & 2.6 \\
\hline $\mathrm{S} 270$ & 3 & 0.8 & 2.8 & 22.0 & 3.5 & 1.0 & 1.5 & 22.1 & 1.5 & 1.1 & 5.3 & 23.7 & 4.5 \\
\hline $\mathrm{S} 283$ & 1 & 0.4 & 0.5 & 52.2 & 1.2 & $\leq 0.5$ & - & - & - & $<0.5$ & - & - & - \\
\hline $\mathrm{S} 284$ & 3 & $\leq 0.3$ & - & - & - & $<0.4$ & - & - & - & $<0.8$ & - & - & - \\
\hline $212.25-1.10$ & 1 & 3.4 & 8.3 & 41.6 & 2.3 & 2.7 & 8.9 & 41.5 & 3.1 & 3.9 & 11.8 & 41.8 & 2.8 \\
\hline MON R2 & 1 & 9.5 & 22.7 & 10.8 & 2.3 & 6.6 & 25.2 & 10.7 & 3.6 & 8.2 & 21.8 & 10.6 & 2.5 \\
\hline MON R2(IRS3) & 1 & 10.7 & 32.7 & 10.3 & 2.9 & 7.8 & 37.2 & 10.1 & 4.5 & 10.1 & 34.9 & 10.0 & 3.2 \\
\hline S286 & 1 & 1.7 & 7.8 & 50.4 & 4.4 & 1.3 & 8.0 & 50.5 & 5.7 & $\leq 0.5$ & - & - & - \\
\hline S305 & 1 & 4.1 & 8.7 & 42.4 & 2.0 & 1.7 & 5.3 & 42.3 & 2.9 & $<1.2$ & - & - & - \\
\hline BBW 33 & 3 & $<0.3$ & - & - & - & $<0.7$ & - & - & - & $<1.5$ & - & - & - \\
\hline
\end{tabular}

1 - Data from June 1990.

2 - Data from April 1991.

3 - Data from Oct 1991. 


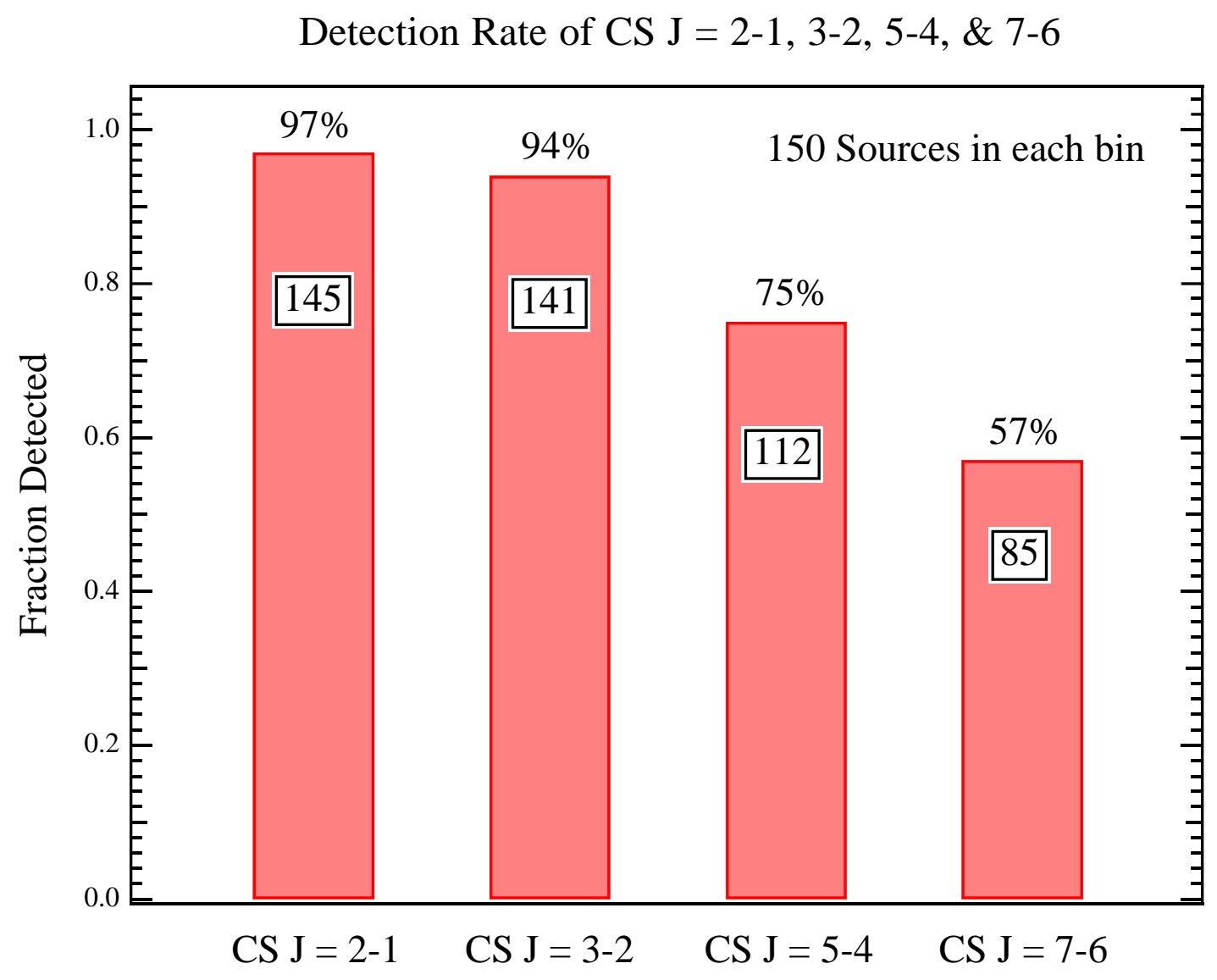


Table3

$\mathrm{C}^{34}$ S Source List

\begin{tabular}{|c|c|c|c|c|c|c|c|c|c|c|c|c|c|c|c|c|c|}
\hline \multirow{3}{*}{$\begin{array}{l}\text { Source } \\
\text { Name }\end{array}$} & \multirow{3}{*}{ Flag } & \multicolumn{4}{|c|}{$\mathrm{C}^{34} \mathrm{~S} \mathrm{~J}=2-1$} & \multicolumn{4}{|c|}{$\mathrm{C}^{34} \mathrm{~S} \mathrm{~J}=3-2$} & \multicolumn{4}{|c|}{$\mathrm{C}^{34} \mathrm{~S} \mathrm{~J}=5-4$} & \multicolumn{4}{|c|}{$\mathrm{C}^{34} \mathrm{~S} J=7-6$} \\
\hline & & $\mathbf{T}_{\mathbf{R}}{ }^{*}$ & $\int T_{R}{ }^{*} d V$ & $\mathbf{V}_{\text {LSR }}$ & FWHM & $\mathbf{T}_{\mathbf{R}}{ }^{*}$ & $\int T_{\mathbf{R}}{ }^{*} \mathrm{dV}$ & $\mathbf{V}_{\text {LSR }}$ & FWHM & $\mathbf{T}_{\mathbf{R}}{ }^{*}$ & $\int \mathbf{T}_{\mathbf{R}}{ }^{*} \mathrm{dV}$ & $\mathbf{V}_{\text {LSR }}$ & FWHM & $\mathbf{T}_{\mathbf{R}}{ }^{*}$ & $\int T_{\mathbf{R}}{ }^{*} \mathrm{dV}$ & $\mathbf{V}_{\text {LSR }}$ & FWHM \\
\hline & & $\mathbf{K}$ & $\mathrm{K} \mathrm{km} \mathrm{s}^{-1}$ & $\mathrm{~km} \mathrm{~s}^{-1}$ & $\mathrm{~km} \mathrm{~s}^{-1}$ & $\mathbf{K}$ & $\mathrm{K} \mathrm{km} \mathrm{s}^{-1}$ & $\mathrm{~km} \mathrm{~s}^{-1}$ & $\mathrm{~km} \mathrm{~s}^{-1}$ & $\mathbf{K}$ & $\mathrm{K} \mathrm{km} \mathrm{s}^{-1}$ & $\mathrm{~km} \mathrm{~s}^{-1}$ & $\mathrm{~km} \mathrm{~s}^{-1}$ & $\mathbf{K}$ & $\mathrm{K} \mathrm{km} \mathrm{s}^{-1}$ & $\mathrm{~km} \mathrm{~s}^{-1}$ & $\mathrm{~km} \mathrm{~s}^{-1}$ \\
\hline W28 A2(2) & 3 & 3.3 & 12.1 & 9.3 & 3.4 & 5.1 & 21.8 & 9.3 & 4.0 & 2.5 & 11.2 & 9.3 & 4.2 & - & - & - & - \\
\hline W28 A2(1) & 2 & 5.8 & 27.6 & 9.6 & 4.5 & 6.4 & 37.2 & 9.6 & 5.5 & - & - & - & - & 3.0 & 22.2 & 10.0 & 7.0 \\
\hline M8 E & 2 & 4.8 & 10.7 & 10.9 & 2.1 & 4.0 & 10.2 & 11.0 & 2.4 & $<0.9$ & - & - & - & 1.0 & 3.7 & 11.4 & 3.4 \\
\hline $9.62+0.10$ & 3 & (3.0) & 19.8 & 4.6 & 6.6 & $(4.5)$ & 33.4 & 4.3 & 7.4 & (3.7) & 29.8 & 4.8 & 8.0 & - & - & - & - \\
\hline W31(1) & 3 & (2.9) & 22.9 & 66.6 & 7.8 & (3.9) & 33.8 & 66.5 & 8.6 & (3.8) & 29.4 & 67.2 & 7.8 & - & - & - & - \\
\hline $10.60-0.40$ & 2 & (8.2) & 53.3 & -2.9 & 6.5 & (11.4) & 81.0 & -2.9 & 7.1 & $<2.0$ & - & - & - & (5.9) & 47.2 & -1.9 & 8.0 \\
\hline W33 B & 3 & 0.8 & 4.3 & 55.4 & 5.0 & 1.1 & 4.7 & 55.9 & 4.2 & 0.9 & 5.9 & 55.8 & 6.0 & - & - & - & - \\
\hline W33 Cont C1 & 2 & 2.4 & 6.3 & 33.5 & 2.5 & - & - & - & - & - & - & - & - & - & - & - & - \\
\hline W33 Cont C2 & 2 & 4.7 & 15.1 & 36.1 & 3.0 & 3.7 & 24.1 & 35.6 & 6.2 & - & - & - & - & 2.4 & 13.0 & 35.6 & 5.0 \\
\hline W33 Cont C3 & 2 & 2.3 & 6.1 & 39.6 & 2.5 & - & - & - & - & - & - & - & - & - & - & - & - \\
\hline $12.89+0.49$ & 3 & 2.7 & 10.5 & 33.3 & 3.7 & 3.6 & 15.3 & 33.2 & 4.0 & 2.6 & 8.3 & 33.2 & 3.0 & - & - & - & - \\
\hline $14.33-0.64$ & 2 & 3.1 & 10.0 & 22.1 & 3.0 & 3.9 & 13.8 & 22.0 & 3.4 & - & - & - & - & 1.5 & 6.5 & 22.3 & 3.9 \\
\hline M17(6) & 3 & 1.3 & 2.9 & 21.0 & 2.2 & 2.9 & 6.4 & 21.0 & 2.1 & $\leq 0.5$ & - & - & - & - & - & - & - \\
\hline $19.61-0.23$ & 3 & 1.5 & 11.5 & 42.3 & 7.2 & 1.9 & 15.4 & 41.6 & 7.5 & $<0.5$ & - & - & - & - & - & - & - \\
\hline $23.96+0.16$ & 3 & 2.4 & 6.4 & 80.4 & 2.6 & 3.4 & 8.5 & 80.5 & 2.4 & 1.0 & 4.9 & 80.1 & 4.8 & - & - & - & - \\
\hline W42 & 3 & (2.7) & 19.8 & 110.7 & 7.4 & (3.8) & 28.6 & 110.8 & 7.6 & $(3.2)$ & 30.3 & 110.6 & 9.5 & - & - & - & - \\
\hline W43 S & 1 & - & - & - & - & 5.6 & 22.9 & 97.8 & 3.9 & 5.2 & 26.2 & 98.1 & 4.7 & 1.5 & 7.2 & 98.3 & 4.6 \\
\hline W43 Main $1 \mathrm{C} 1$ & 3 & 0.7 & 3.3 & 91.3 & 4.5 & 0.5 & 4.0 & 91.4 & 7.4 & $<0.5$ & - & - & - & - & - & - & - \\
\hline W43 Main 1 C2 & 3 & 0.5 & 1.1 & 97.8 & 1.8 & - & - & - & - & $<0.5$ & - & - & - & - & - & - & - \\
\hline W43 Main 3 & 3 & (1.4) & 11.6 & 99.0 & 8.4 & (1.9) & 16.5 & 99.0 & 8.7 & $(1.5)$ & 10.2 & 100.5 & 6.7 & - & - & - & - \\
\hline W43 Main 4 & 3 & $<0.4$ & - & - & - & $<0.5$ & - & - & - & $<0.2$ & - & - & - & - & - & - & - \\
\hline $31.44-0.26$ & 1 & - & - & - & - & 3.2 & 14.4 & 87.2 & 4.3 & 2.5 & 14.8 & 87.4 & 5.6 & - & - & - & - \\
\hline $31.41+0.31$ & 1 & - & - & - & - & $(2.3)$ & 15.9 & 97.1 & 6.9 & (4.4) & 26.1 & 97.3 & 6.0 & - & - & - & - \\
\hline $32.8+0.20 \mathrm{~A}$ & 1 & - & - & - & - & 1.1 & 8.9 & 13.8 & 8.0 & 1.1 & 9.2 & 14.5 & 7.6 & - & - & - & - \\
\hline W44 & 1 & - & - & - & - & (6.9) & 44.1 & 58.0 & 6.4 & (6.5) & 48.6 & 58.5 & 7.5 & (3.1) & 30.5 & 58.4 & 9.7 \\
\hline $35.58-0.03$ & 2 & 1.2 & 6.3 & 53.3 & 5.2 & 0.9 & 6.3 & 52.4 & 6.4 & $\leq 0.2$ & - & - & - & - & - & - & - \\
\hline W49 N C1 & 2 & 1.0 & 9.2 & 4.2 & 8.8 & 2.2 & 13.7 & 3.6 & 5.8 & - & - & - & - & 1.5 & 15.7 & 5.2 & 9.8 \\
\hline W49 N C2 & 2 & 2.0 & 13.1 & 12.2 & 6.1 & 2.0 & 14.6 & 12.1 & 6.9 & - & - & - & - & 0.8 & 5.6 & 14.2 & 7.0 \\
\hline
\end{tabular}


Table 3

$\mathrm{C}^{34} \mathrm{~S}$ Source List (Continued)

\begin{tabular}{|c|c|c|c|c|c|c|c|c|c|c|c|c|c|c|c|c|c|}
\hline \multirow[b]{2}{*}{$\begin{array}{l}\text { Source } \\
\text { Name }\end{array}$} & \multirow[b]{2}{*}{ Flag } & \multicolumn{4}{|c|}{$\mathrm{C}^{34} \mathrm{~S} J=2-1$} & \multicolumn{4}{|c|}{$\mathrm{C}^{34} \mathrm{~S} \mathrm{~J}=3-2$} & \multicolumn{4}{|c|}{$C^{34} S$ J $=5-4$} & \multicolumn{4}{|c|}{$C^{34} S J=7-6$} \\
\hline & & $\begin{array}{c}\mathbf{T}_{\mathbf{R}}^{*} \\
\mathbf{K}\end{array}$ & $\begin{array}{r}\int T_{R}{ }^{*} \mathrm{dV} \\
\mathrm{K} \mathrm{km} \mathrm{s}^{-1} \\
\end{array}$ & $\begin{array}{r}\text { VLSR } \\
\mathrm{km} \mathrm{s}^{-1} \\
\end{array}$ & $\begin{array}{c}\text { FWHM } \\
\mathrm{km} \mathrm{s}^{-1} \\
\end{array}$ & $\begin{array}{c}\mathbf{T}_{\mathbf{R}}^{*} \\
\mathbf{K} \\
\end{array}$ & $\begin{array}{r}\int T_{R}{ }^{*} \mathrm{dV} \\
\mathrm{K} \mathrm{km} \mathrm{s}^{-1} \\
\end{array}$ & $\begin{array}{r}\text { VISR } \\
\mathrm{km} \mathrm{s}^{\mathbf{- 1}} \\
\end{array}$ & $\begin{array}{c}\text { FWHM } \\
\mathrm{km} \mathrm{s}^{-1} \\
\end{array}$ & $\begin{array}{c}\mathbf{T}_{\mathbf{R}}^{*} \\
\mathbf{K}\end{array}$ & $\begin{array}{r}\int T_{R}{ }^{*} \mathrm{dV} \\
\mathrm{K} \mathrm{km} \mathrm{s}^{-1} \\
\end{array}$ & $\begin{array}{r}\text { VLSR } \\
\mathrm{km} \mathrm{s}^{-1} \\
\end{array}$ & $\begin{array}{c}\text { FWHM } \\
\mathrm{km} \mathrm{s}^{-1} \\
\end{array}$ & $\begin{array}{c}\mathbf{T}_{\mathbf{R}}^{*} \\
\mathbf{K}\end{array}$ & $\begin{array}{r}\int T_{R}{ }^{*} \mathrm{dV} \\
\mathrm{K} \mathrm{km} \mathrm{s}^{-1} \\
\end{array}$ & $\begin{array}{r}\text { VLSR } \\
\mathrm{km} \mathrm{s}^{\mathbf{- 1}} \\
\end{array}$ & $\begin{array}{c}\text { FWHM } \\
\mathrm{km} \mathrm{s}^{-1} \\
\end{array}$ \\
\hline $\mathrm{OH} 43.8-0.10$ & 2 & 1.5 & 8.2 & 44.2 & 5.3 & $\leq 0.3$ & - & - & - & $<0.4$ & - & - & - & - & - & - & - \\
\hline W51W & 1 & - & - & - & - & 2.0 & 6.6 & 50.9 & 3.1 & 1.5 & 5.4 & 50.9 & 3.5 & - & - & - & - \\
\hline W51 N C1 & 1 & - & - & - & - & 0.5 & 1.4 & 49.7 & 2.9 & - & - & - & - & - & - & - & - \\
\hline W51 N C2 & 1 & - & - & - & - & 3.3 & 26.5 & 60.8 & 7.5 & 3.3 & 31.0 & 60.8 & 8.8 & 2.6 & 23.7 & 60.1 & 8.6 \\
\hline W51 M & 1 & - & - & - & - & 9.6 & 90.7 & 56.6 & 8.9 & 10.5 & 101.0 & 57.5 & 9.1 & 5.5 & 57.2 & 56.2 & 9.8 \\
\hline $\mathrm{K} 3-50$ & 3 & 0.4 & 3.3 & -23.2 & 8.0 & 0.7 & 3.8 & -24.0 & 5.4 & $<0.3$ & - & - & - & - & - & - & - \\
\hline ON 3 & 3 & $(0.6)$ & 3.3 & -21.8 & 5.3 & $(1.5)$ & 3.1 & -24.6 & 2.1 & $<0.4$ & - & - & - & - & - & - & - \\
\hline ON $2 \mathrm{~S}$ & 1 & - & - & - & - & $(2.4)$ & 10.0 & -1.6 & 4.1 & $(3.1)$ & 6.8 & -1.4 & 2.2 & - & - & - & - \\
\hline CRL 2591 & 2 & 1.7 & 5.8 & -6.2 & 3.3 & 1.5 & 5.0 & -6.1 & 3.2 & $<0.3$ & - & - & - & - & - & - & - \\
\hline DR $21 \mathrm{~S}$ & 1 & - & - & - & - & 2.4 & 11.0 & -2.3 & 4.4 & 1.9 & 9.9 & -2.3 & 4.9 & - & - & - & - \\
\hline $\mathrm{W} 75(\mathrm{OH})$ & 1 & - & - & - & - & $(4.8)$ & 23.9 & -2.8 & 5.0 & $(5.6)$ & 27.4 & -2.6 & 4.9 & (1.9) & 12.0 & -3.0 & 6.3 \\
\hline W75 N & 1 & - & - & - & - & 2.6 & 11.9 & 9.2 & 4.3 & 1.9 & 9.7 & 9.7 & 4.8 & 1.1 & 7.6 & 9.9 & 6.5 \\
\hline Cep A & 2 & $(1.3)$ & 2.7 & -10.4 & 2.1 & $(1.0)$ & 1.9 & -11.4 & 2.0 & $<0.3$ & - & - & - & - & - & - & - \\
\hline S157 & 1 & 0.3 & 1.3 & -44.4 & 4.0 & 0.8 & 2.2 & -44.1 & 2.6 & $\leq 0.3$ & - & - & - & - & - & - & - \\
\hline S158 & 1 & $(2.6)$ & 8.8 & -56.0 & 3.4 & $(3.8)$ & 18.2 & -55.7 & 4.8 & $(3.9)$ & 16.2 & -55.5 & 4.2 & - & - & - & - \\
\hline S158 A & 1 & 1.3 & 6.8 & -57.2 & 4.9 & 2.5 & 11.4 & -57.5 & 4.3 & 2.3 & 8.0 & -58.0 & 3.3 & 1.3 & 3.2 & -57.1 & 2.3 \\
\hline IRAS $00338+6312$ & 1 & $(0.8)$ & 3.5 & -17.7 & 4.4 & $(1.4)$ & 5.0 & -17.7 & 3.6 & $(1.2)$ & 2.9 & -17.5 & 2.4 & - & - & - & - \\
\hline NGC 281 & 1 & 0.6 & 3.1 & -30.4 & 4.8 & 1.6 & 6.6 & -30.7 & 4.0 & 0.7 & 3.6 & -30.7 & 5.0 & - & - & - & - \\
\hline W3(2) & 1 & - & - & - & - & $(1.3)$ & 3.9 & -38.9 & 3.1 & $(1.5)$ & 4.2 & -38.1 & 2.8 & - & - & - & - \\
\hline $\mathrm{W} 3(\mathrm{OH})$ & 1 & - & - & - & - & $(5.1)$ & 27.2 & -47.2 & 5.3 & $(8.0)$ & 46.6 & -47.1 & 5.8 & - & - & - & - \\
\hline IRAS 02461+6147 & 1 & 0.2 & 0.7 & -42.1 & 3.0 & 0.9 & 2.0 & -42.2 & 2.0 & $<0.4$ & - & - & - & - & - & - & - \\
\hline IRAS $02541+6208$ & 1 & $\leq 0.2$ & - & - & - & 0.4 & 1.2 & -52.1 & 2.9 & $\leq 0.3$ & - & - & - & - & - & - & - \\
\hline S235 & 1 & 2.5 & 4.8 & -16.9 & 1.8 & 3.8 & 9.1 & -17.1 & 2.2 & 1.9 & 5.2 & -17.2 & 2.5 & - & - & - & - \\
\hline S255/7 & 1 & 2.8 & 6.5 & 7.3 & 2.2 & 3.6 & 9.7 & 7.3 & 2.6 & 1.7 & 5.4 & 7.8 & 3.0 & - & - & - & - \\
\hline MON R2 & 1 & 0.9 & 1.4 & 11.1 & 1.4 & 1.3 & 2.0 & 10.8 & 1.5 & $<0.4$ & - & - & - & - & - & - & - \\
\hline MON R2(IRS3) & 1 & 0.7 & 2.6 & 10.6 & 3.3 & 0.9 & 2.5 & 10.4 & 2.5 & 0.8 & 2.8 & 10.6 & 3.2 & - & - & - & - \\
\hline
\end{tabular}

1 - Data from June 1990

2 - Data from April 1991

3 - Data from Oct 1991 

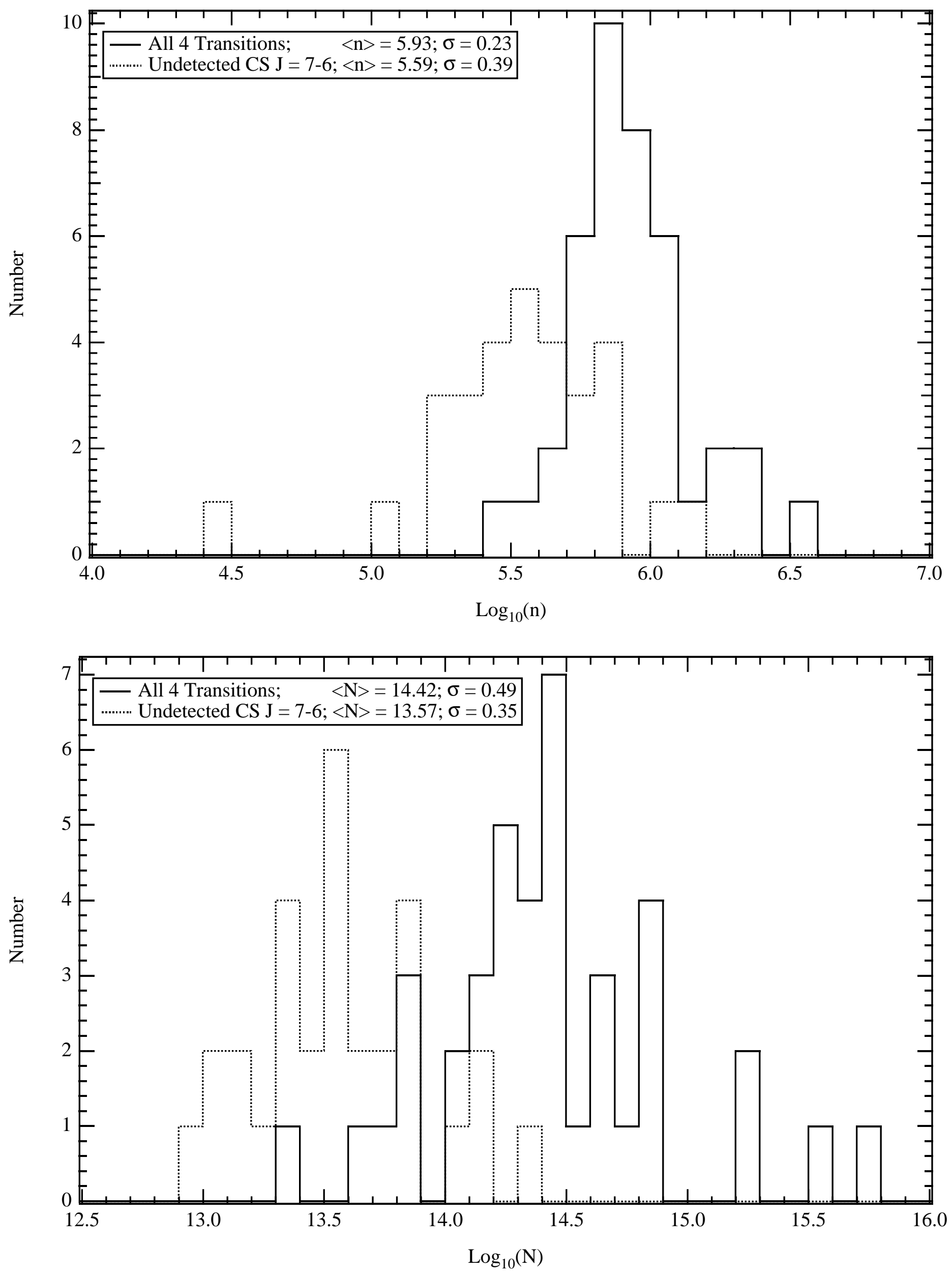

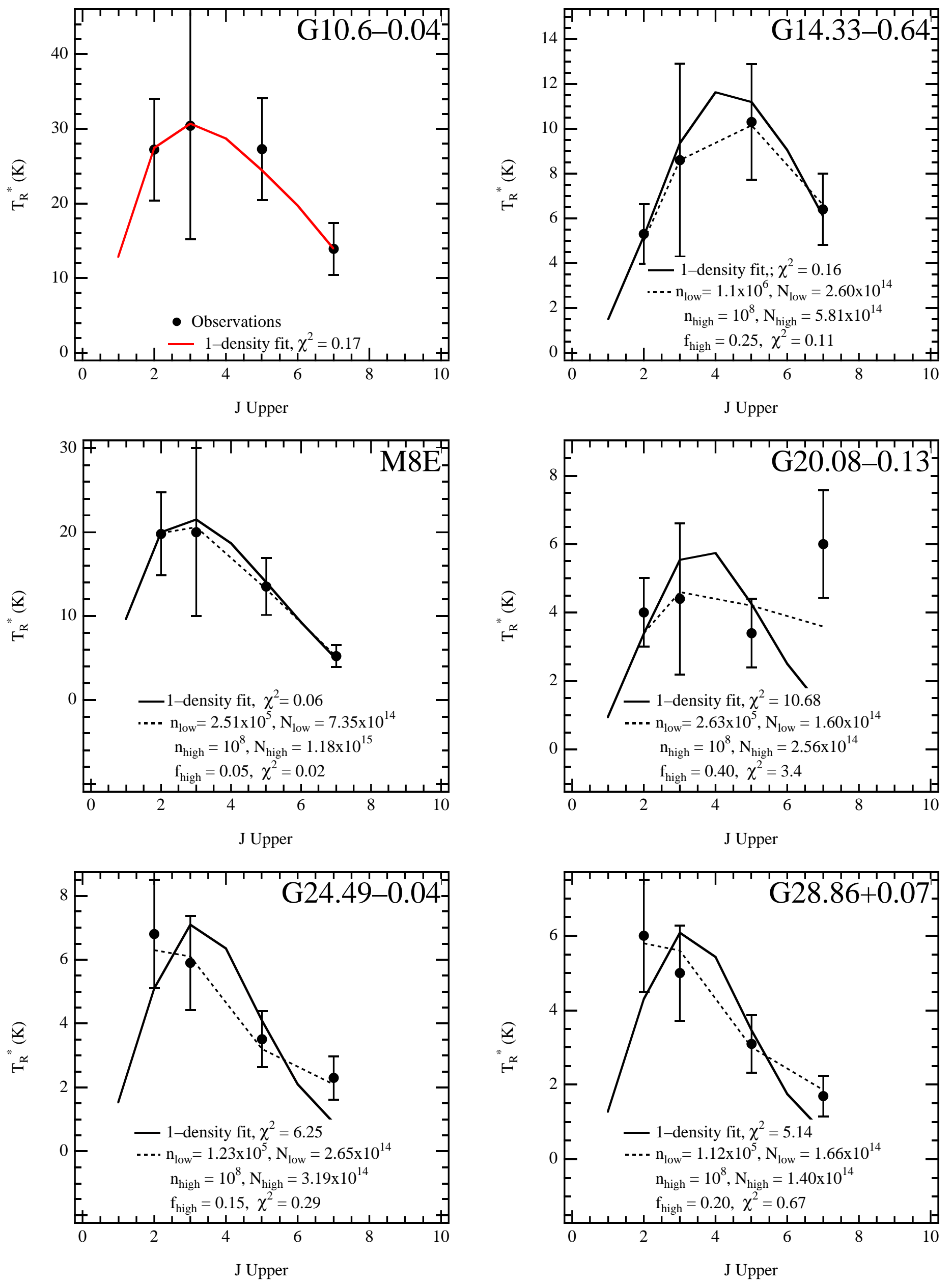

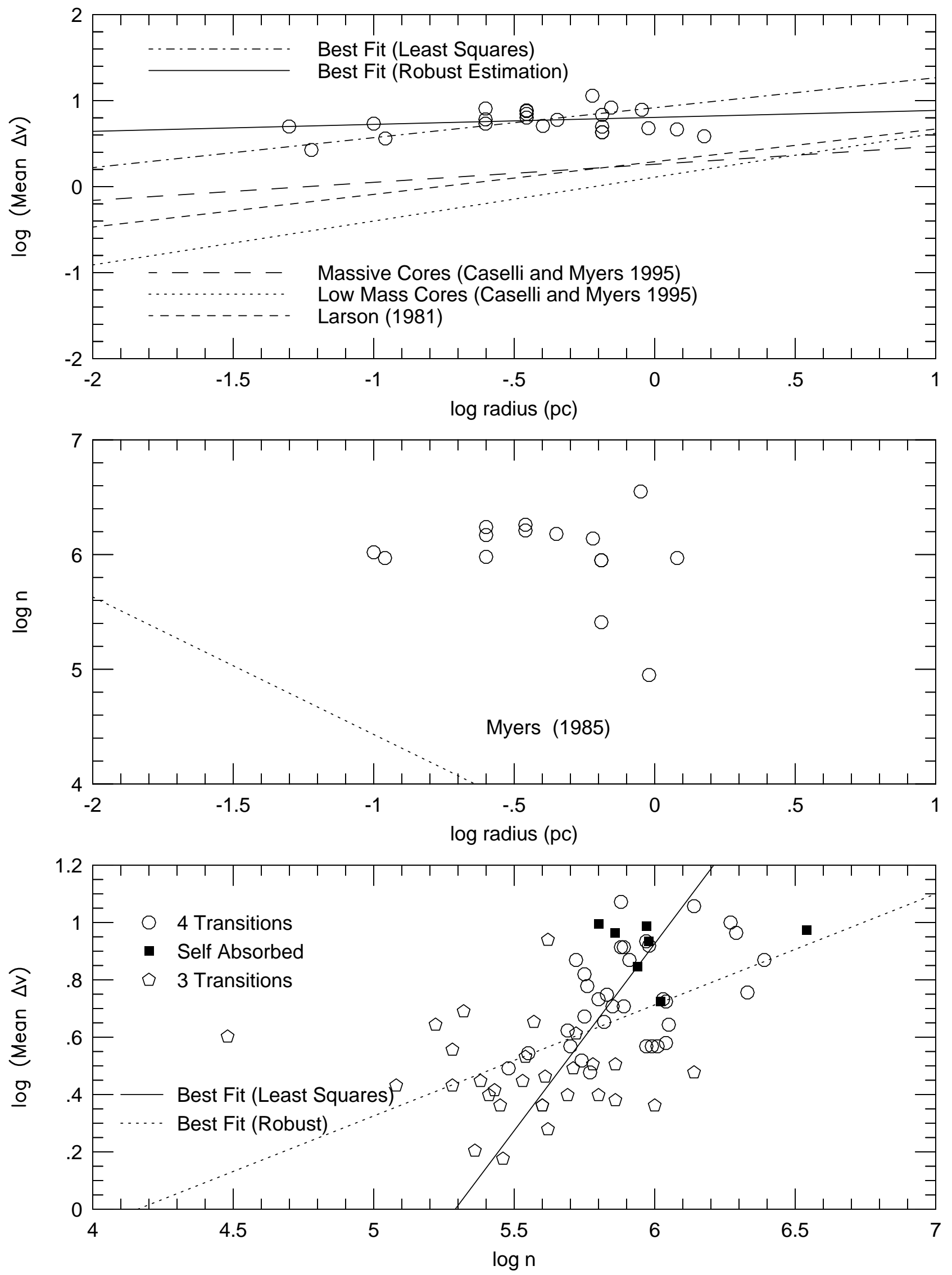
Table 6

Source Densities

\begin{tabular}{|c|c|c|c|c|c|c|c|c|c|c|c|c|}
\hline Source & $\begin{array}{c}\text { Flag } \\
\text { CS }\end{array}$ & $\begin{array}{c}\log (\mathbf{n}) \\
\mathrm{CS}\end{array}$ & $\begin{array}{c}\log (\mathbf{N}) \\
\mathrm{CS}\end{array}$ & $\begin{array}{l}\chi^{2} \\
\text { CS }\end{array}$ & $\begin{array}{l}\text { Flag } \\
\mathrm{C}^{34} \mathrm{~S}\end{array}$ & $\begin{array}{c}\log (n) \\
C^{34} S\end{array}$ & $\begin{array}{c}\log (N) \\
C^{34} S\end{array}$ & $\begin{array}{c}\chi^{2} \\
c^{34} s\end{array}$ & $\begin{array}{r}\text { Flag } \\
\text { both }\end{array}$ & $\begin{array}{c}\log (\mathbf{n}) \\
\text { both }\end{array}$ & $\begin{array}{c}\log (\mathbf{N}) \\
\text { both }\end{array}$ & $\begin{array}{c}\chi^{2} \\
\text { both }\end{array}$ \\
\hline RCW 142 & $\bar{a}$ & $5.88 \pm 0.27$ & $15.23 \pm 1.33$ & 0.87 & - & - & - & - & - & - & - & - \\
\hline W28 A2(2) & $\mathrm{a}$ & $5.85 \pm 0.26$ & $14.78 \pm 0.82$ & 3.62 & $\mathrm{~d}$ & $5.70 \pm 0.18$ & $13.83 \pm 0.11$ & 0.08 & $\mathrm{i}$ & $5.69 \pm 0.14$ & $14.91 \pm 0.33$ & 4.65 \\
\hline W28 A2(1) & $\mathrm{a}$ & $5.88 \pm 0.38$ & $15.74 \pm 3.74$ & 0.52 & - & - & - & - & - & - & - & - \\
\hline M8E & $\mathrm{a}$ & $5.55 \pm 0.29$ & $14.84 \pm 0.88$ & 0.06 & - & - & - & - & - & - & - & - \\
\hline $9.62+0.10$ & $\mathrm{a}, \mathrm{sa}$ & $5.97 \pm 0.17$ & $14.86 \pm 0.99$ & 0.51 & $\mathrm{~d}$ & $5.95 \pm 0.21$ & $14.10 \pm 0.14$ & 0.08 & $\mathrm{i}$ & $5.80 \pm 0.13$ & $15.05 \pm 0.52$ & 8.96 \\
\hline W31(1) & $\mathrm{a}, \mathrm{sa}$ & $5.86 \pm 0.16$ & $14.83 \pm 0.91$ & 2.08 & $\mathrm{~d}$ & $6.01 \pm 0.23$ & $14.11 \pm 0.03$ & 0.45 & $\mathrm{i}$ & $5.68 \pm 0.13$ & $15.01 \pm 0.63$ & 11.1 \\
\hline $10.60-0.40$ & $\mathrm{a}, \mathrm{sa}$ & $5.80 \pm 0.32$ & $15.56 \pm 2.44$ & 0.17 & - & - & - & - & - & - & - & - \\
\hline $12.21-0.10$ & $\mathrm{a}$ & $5.91 \pm 0.17$ & $14.47 \pm 0.56$ & 1.40 & - & - & - & - & - & - & - & - \\
\hline $12.42+0.50$ & $\mathrm{a}$ & $5.74 \pm 0.18$ & $14.31 \pm 0.41$ & 1.29 & - & - & - & - & - & - & - & - \\
\hline W33 B & $\mathrm{a}$ & $5.99 \pm 0.14$ & $13.85 \pm 0.31$ & 1.19 & d & $5.93 \pm 0.27$ & $13.29 \pm 0.10$ & 0.24 & $\mathrm{i}$ & $5.89 \pm 0.15$ & $13.98 \pm 0.22$ & 6.58 \\
\hline W33 Cont C1 & $\mathrm{b}$ & $5.72 \pm 0.22$ & $14.36 \pm 0.30$ & 0.01 & - & - & - & - & 1 & $5.54 \pm 0.22$ & $14.47 \pm 0.37$ & 2.46 \\
\hline W33 Cont C2 & $\mathrm{a}$ & $5.89 \pm 0.23$ & $14.69 \pm 0.69$ & 2.89 & - & - & - & - & - & - & - & - \\
\hline W33 Cont C3 & $\mathrm{b}$ & $5.71 \pm 0.45$ & $14.20 \pm 0.55$ & 0.05 & - & - & - & - & 1 & $5.29 \pm 0.39$ & $14.44 \pm 0.42$ & 1.05 \\
\hline $12.89+0.49$ & $\mathrm{a}$ & $6.33 \pm 0.23$ & $14.10 \pm 0.24$ & 2.51 & d & $5.83 \pm 0.20$ & $13.69 \pm 0.08$ & 0.21 & $\mathrm{i}$ & $6.30 \pm 0.18$ & $14.14 \pm 0.09$ & 31.5 \\
\hline $13.87+0.28$ & $\mathrm{a}$ & $6.05 \pm 0.21$ & $14.10 \pm 0.42$ & 4.14 & - & - & - & - & - & - & - & - \\
\hline $14.33-0.64$ & $\mathrm{a}$ & $6.39 \pm 0.19$ & $14.50 \pm 0.35$ & 0.16 & - & - & - & - & - & - & - & - \\
\hline $19.61-0.13$ & $\mathrm{~b}$ & $5.57 \pm 0.21$ & $13.58 \pm 0.33$ & 2.47 & - & - & - & - & - & - & - & - \\
\hline $19.61-0.23$ & $\mathrm{a}$ & $6.29 \pm 0.19$ & $14.46 \pm 0.39$ & 2.94 & e & $5.53 \pm 0.45$ & $13.69 \pm 0.39$ & 0.00 & $\mathrm{j}$ & $6.11 \pm 0.19$ & $14.54 \pm 0.36$ & 11.5 \\
\hline $20.08-0.13$ & $\mathrm{a}$ & $5.97 \pm 0.29$ & $14.24 \pm 0.43$ & 10.7 & - & - & - & - & - & - & - & - \\
\hline $22.36+0.07$ & $\mathrm{~b}$ & $5.78 \pm 0.24$ & $13.58 \pm 0.16$ & 2.64 & - & - & - & - & - & - & - & - \\
\hline $24.49-0.04$ & $\mathrm{a}$ & $5.75 \pm 0.22$ & $14.26 \pm 0.64$ & 6.25 & - & - & - & - & - & - & - & - \\
\hline W42 & $\mathrm{a}, \mathrm{sa}$ & $6.54 \pm 0.37$ & $14.41 \pm 0.37$ & 3.23 & - & - & - & - & - & - & - & - \\
\hline $28.83-0.25$ & $\mathrm{a}$ & $6.04 \pm 0.29$ & $13.31 \pm 0.15$ & 6.25 & - & - & - & - & - & - & - & - \\
\hline $28.86+0.07$ & $\mathrm{a}$ & $5.75 \pm 0.21$ & $14.03 \pm 0.44$ & 5.14 & - & - & - & - & - & - & - & - \\
\hline
\end{tabular}


Table 6 (Continued)

Source Density

\begin{tabular}{|c|c|c|c|c|c|c|c|c|c|c|c|c|}
\hline Source & $\begin{array}{c}\text { Flag } \\
\text { CS }\end{array}$ & $\begin{array}{c}\log (n) \\
\text { CS }\end{array}$ & $\begin{array}{c}\log (\mathbf{N}) \\
\mathrm{CS}\end{array}$ & $\begin{array}{l}\chi^{2} \\
\text { CS }\end{array}$ & $\begin{array}{l}\text { Flag } \\
C^{34} S\end{array}$ & $\begin{array}{c}\log (n) \\
C^{34} S \\
\end{array}$ & $\begin{array}{c}\log (N) \\
C^{34} S\end{array}$ & $\begin{array}{c}\chi^{2} \\
c^{34} s\end{array}$ & $\begin{array}{l}\text { Flag } \\
\text { both }\end{array}$ & $\begin{array}{c}\log (n) \\
\text { both }\end{array}$ & $\begin{array}{c}\log (\mathbf{N}) \\
\text { both }\end{array}$ & $\begin{array}{c}\chi^{2} \\
\text { both }\end{array}$ \\
\hline $29.95-0.01$ & $\mathrm{~b}$ & $5.28 \pm 0.20$ & $13.80 \pm 0.37$ & 0.17 & - & - & - & - & - & - & - & - \\
\hline W43 Main 2 & $\mathrm{~b}, \mathrm{sa}$ & $5.82 \pm 0.21$ & $14.04 \pm 0.31$ & 0.50 & - & - & - & - & - & - & - & - \\
\hline $32.05+0.06$ & $\mathrm{a}, \mathrm{sa}$ & $5.98 \pm 0.17$ & $14.24 \pm 0.58$ & 2.54 & - & - & - & - & - & - & - & - \\
\hline $32.74-0.08 \mathrm{~A}$ & $\mathrm{a}$ & $5.98 \pm 0.30$ & $13.87 \pm 0.39$ & 6.03 & - & - & - & - & - & - & - & - \\
\hline $32.80+0.20 \mathrm{~B}$ & $\mathrm{~b}$ & $5.62 \pm 0.28$ & $14.18 \pm 0.56$ & 1.78 & - & - & - & - & - & - & - & - \\
\hline $33.81-0.19$ & $\mathrm{a}$ & $6.01 \pm 0.17$ & $13.77 \pm 0.12$ & 1.89 & - & - & - & - & - & - & - & - \\
\hline $35.20-0.74$ & $\mathrm{a}, \mathrm{sa}$ & $5.94 \pm 0.20$ & $14.44 \pm 0.67$ & 2.86 & - & - & - & - & - & - & - & - \\
\hline $35.58-0.03$ & $\mathrm{a}$ & $6.03 \pm 0.16$ & $14.30 \pm 0.45$ & 0.90 & $\mathrm{e}$ & $4.99 \pm 0.65$ & $13.47 \pm 0.99$ & 0.00 & $\mathrm{j}$ & $5.96 \pm 0.15$ & $14.35 \pm 0.35$ & 3.87 \\
\hline S76 E & $\mathrm{a}$ & $5.70 \pm 0.20$ & $14.64 \pm 0.58$ & 0.02 & - & - & - & - & - & - & - & - \\
\hline $40.62-0.14$ & $\mathrm{~b}$ & $5.32 \pm 0.19$ & $13.86 \pm 0.53$ & 0.01 & - & - & - & - & - & - & - & - \\
\hline W49 S & $\mathrm{a}$ & $5.89 \pm 0.15$ & $14.38 \pm 0.28$ & 0.05 & - & - & - & - & - & - & - & - \\
\hline W49 N C1 & $\mathrm{a}$ & $6.27 \pm 0.21$ & $14.83 \pm 0.59$ & 0.98 & - & - & - & - & - & - & - & - \\
\hline W49 N C2 & $\mathrm{a}$ & $5.72 \pm 0.22$ & $14.64 \pm 1.04$ & 3.70 & - & - & - & - & - & - & - & - \\
\hline $\mathrm{OH} 43.8-0.10$ & $\mathrm{a}$ & $5.76 \pm 0.15$ & $14.40 \pm 0.61$ & 0.17 & - & - & - & - & $\mathrm{k}$ & $5.65 \pm 0.15$ & $14.48 \pm 0.29$ & 1.86 \\
\hline $45.07+0.13$ & $\mathrm{a}$ & $6.04 \pm 0.21$ & $14.28 \pm 0.45$ & 3.37 & - & - & - & - & - & - & - & - \\
\hline $48.61+0.02$ & $\mathrm{a}$ & $5.83 \pm 0.19$ & $14.05 \pm 0.45$ & 2.79 & - & - & - & - & - & - & - & - \\
\hline W51 M & $\mathrm{a}$ & $6.14 \pm 0.24$ & $15.22 \pm 1.45$ & 3.09 & $\mathrm{f}$ & $6.29 \pm 0.20$ & $14.60 \pm 0.79$ & 0.01 & - & - & - & - \\
\hline $59.78+0.06$ & $\mathrm{a}$ & $5.48 \pm 0.22$ & $14.13 \pm 0.46$ & 2.85 & - & - & - & - & - & - & - & - \\
\hline S87 & $\mathrm{a}$ & $5.69 \pm 0.19$ & $14.46 \pm 0.53$ & 0.47 & - & - & - & - & - & - & - & - \\
\hline S88 B & $\mathrm{b}$ & $5.61 \pm 0.16$ & $13.77 \pm 0.15$ & 0.52 & - & - & - & - & - & - & - & - \\
\hline CRL 2591 & $\mathrm{a}$ & $5.97 \pm 0.18$ & $14.45 \pm 0.36$ & 0.58 & $\mathrm{e}$ & $5.15 \pm 0.46$ & $13.37 \pm 0.35$ & 0.00 & $\mathrm{j}$ & $6.01 \pm 0.14$ & $14.40 \pm 0.19$ & 3.51 \\
\hline IRAS $21519+5613$ & $\mathrm{a}$ & $5.80 \pm 0.16$ & $13.88 \pm 0.09$ & 0.17 & - & - & - & - & - & - & - & - \\
\hline IRAS $21512+5625$ & $\mathrm{~b}$ & $5.86 \pm 0.24$ & $12.96 \pm 0.09$ & 0.18 & - & - & - & - & - & - & - & - \\
\hline IRAS $22142+5206$ & $\mathrm{~b}$ & $5.45 \pm 0.18$ & $13.16 \pm 0.18$ & 0.26 & - & - & - & - & - & - & - & - \\
\hline BFS 10 & $\mathrm{~b}$ & $5.69 \pm 0.19$ & $13.44 \pm 0.10$ & 0.73 & - & - & - & - & - & - & - & - \\
\hline IRAS $21561+5806$ & $\mathrm{~b}$ & $5.28 \pm 0.25$ & $13.40 \pm 0.26$ & 0.11 & - & - & - & - & - & - & - & - \\
\hline
\end{tabular}


Table 6 (Continued)

Source Density

\begin{tabular}{|c|c|c|c|c|c|c|c|c|c|c|c|c|}
\hline Source & $\begin{array}{c}\text { Flag } \\
\text { CS }\end{array}$ & $\begin{array}{c}\log (n) \\
\text { CS }\end{array}$ & $\begin{array}{c}\log (\mathbf{N}) \\
\mathrm{CS}\end{array}$ & $\begin{array}{l}\chi^{2} \\
\text { CS }\end{array}$ & $\begin{array}{l}\text { Flag } \\
C^{34} S\end{array}$ & $\begin{array}{c}\log (n) \\
C^{34} S \\
\end{array}$ & $\begin{array}{c}\log (\mathbf{N}) \\
\mathbf{C}^{34} \mathbf{S}\end{array}$ & $\begin{array}{c}\chi^{2} \\
c^{34} s\end{array}$ & $\begin{array}{l}\text { Flag } \\
\text { both }\end{array}$ & $\begin{array}{c}\log (n) \\
\text { both }\end{array}$ & $\begin{array}{c}\log (\mathbf{N}) \\
\text { both }\end{array}$ & $\begin{array}{c}\chi^{2} \\
\text { both }\end{array}$ \\
\hline IRAS $21558+5907$ & $\mathrm{~b}$ & $5.60 \pm 0.22$ & $13.18 \pm 0.13$ & 0.94 & - & - & - & - & - & - & - & - \\
\hline IRAS $22172+5549$ & $\mathrm{~b}$ & $5.38 \pm 0.18$ & $13.62 \pm 0.23$ & 0.04 & - & - & - & - & - & - & - & - \\
\hline IRAS 22134+5834 & $\mathrm{b}$ & $5.60 \pm 0.20$ & $13.54 \pm 0.12$ & 0.25 & - & - & - & - & - & - & - & - \\
\hline IRAS $22305+5803$ & $\mathrm{~b}$ & $6.00 \pm 0.27$ & $13.37 \pm 0.00$ & 0.70 & - & - & - & - & - & - & - & - \\
\hline IRAS $22308+5812$ & $\mathrm{~b}$ & $5.22 \pm 0.31$ & $13.90 \pm 0.69$ & 1.19 & - & - & - & - & - & - & - & - \\
\hline Cep A & $\mathrm{a}, \mathrm{sa}$ & $6.02 \pm 0.15$ & $14.47 \pm 0.33$ & 0.21 & $\mathrm{e}$ & $5.01 \pm 0.45$ & $13.07 \pm 0.50$ & 0.00 & $\mathrm{j}$ & $6.08 \pm 0.13$ & $14.41 \pm 0.26$ & 4.41 \\
\hline $118.96+1.88$ & $\mathrm{~b}$ & $5.43 \pm 0.21$ & $13.55 \pm 0.24$ & 0.64 & - & - & - & - & - & - & - & - \\
\hline IRAS 00117+6412 & $\mathrm{b}$ & $5.53 \pm 0.18$ & $13.57 \pm 0.22$ & 0.23 & - & - & - & - & - & - & - & - \\
\hline IRAS 00211+6549 & $\mathrm{b}$ & $5.54 \pm 0.16$ & $13.70 \pm 0.18$ & 0.26 & - & - & - & - & - & - & - & - \\
\hline IRAS 00379+6248 & $\mathrm{b}$ & $5.46 \pm 0.22$ & $13.59 \pm 0.14$ & 0.25 & - & - & - & - & - & - & - & - \\
\hline IRAS 00468+6527 & $\mathrm{b}$ & $5.80 \pm 0.20$ & $13.23 \pm 0.10$ & 0.58 & - & - & - & - & - & - & - & - \\
\hline IRAS $01045+6505$ & $\mathrm{~b}$ & $5.86 \pm 0.31$ & $13.35 \pm 0.01$ & 1.02 & - & - & - & - & - & - & - & - \\
\hline IRAS 01123+6430 & $\mathrm{b}$ & $5.41 \pm 0.22$ & $13.34 \pm 0.20$ & 0.69 & - & - & - & - & - & - & - & - \\
\hline IRAS 02395+6944 & $\mathrm{a}$ & $5.77 \pm 0.14$ & $13.63 \pm 0.28$ & 0.81 & - & - & - & - & - & - & - & - \\
\hline $140.64+0.67$ & $\mathrm{~b}$ & $5.36 \pm 0.32$ & $13.45 \pm 0.16$ & 0.74 & - & - & - & - & - & - & - & - \\
\hline GL 490 & $\mathrm{~b}$ & $5.08 \pm 0.33$ & $13.74 \pm 0.46$ & 0.03 & - & - & - & - & - & - & - & - \\
\hline S209 & $\mathrm{b}$ & $5.62 \pm 0.60$ & $13.04 \pm 0.15$ & 1.83 & - & - & - & - & - & - & - & - \\
\hline $\mathrm{S} 231$ & $\mathrm{a}$ & $5.82 \pm 0.19$ & $14.27 \pm 0.39$ & 3.12 & - & - & - & - & - & - & - & - \\
\hline S269 B & $\mathrm{b}$ & $4.48 \pm 0.82$ & $13.82 \pm 2.68$ & 2.70 & - & - & - & - & - & - & - & - \\
\hline $\mathrm{S} 270$ & $\mathrm{~b}$ & $6.14 \pm 0.46$ & $13.06 \pm 0.04$ & 0.24 & - & - & - & - & - & - & - & - \\
\hline $212.25-1.10$ & $\mathrm{~b}$ & $6.93 \pm 1.46$ & $13.35 \pm 0.43$ & 3.46 & - & - & - & - & - & - & - & - \\
\hline M17(6) & $\mathrm{c}$ & - & - & - & $\mathrm{e}$ & $7.33 \pm 1.74$ & $13.53 \pm 0.15$ & 0.15 & - & - & - & - \\
\hline W43 S & $\mathrm{c}$ & - & - & - & $\mathrm{f}$ & $6.02 \pm 0.16$ & $13.98 \pm 0.44$ & 0.08 & - & - & - & - \\
\hline W43 Main $1 \mathrm{C} 1$ & $\mathrm{c}$ & - & - & - & $\mathrm{e}$ & $4.95 \pm 0.74$ & $13.23 \pm 2.09$ & 0.00 & - & - & - & - \\
\hline $31.41+0.31$ & $\mathrm{c}, \mathrm{sa}$ & - & - & - & $\mathrm{g}$ & $8.13 \pm 2.48$ & $13.98 \pm 0.00$ & 0.03 & - & - & - & - \\
\hline $31.44-0.26$ & $\mathrm{c}$ & - & - & - & g & $5.97 \pm 0.27$ & $13.74 \pm 0.33$ & 0.00 & - & - & - & - \\
\hline
\end{tabular}


Table 6 (Continued)

Source Density

\begin{tabular}{|c|c|c|c|c|c|c|c|c|c|c|c|c|}
\hline Source & $\begin{array}{c}\text { Flag } \\
\text { CS }\end{array}$ & $\begin{array}{c}\log (\mathbf{n}) \\
\text { CS }\end{array}$ & $\begin{array}{c}\log (\mathbf{N}) \\
\mathrm{CS}\end{array}$ & $\begin{array}{l}\chi^{2} \\
\mathrm{CS}\end{array}$ & $\begin{array}{l}\text { Flag } \\
C^{34} S\end{array}$ & $\begin{array}{c}\log (n) \\
C^{34} S\end{array}$ & $\begin{array}{c}\log (N) \\
C^{34} S\end{array}$ & $\begin{array}{c}\chi^{2} \\
c^{34} s\end{array}$ & $\begin{array}{r}\text { Flag } \\
\text { both }\end{array}$ & $\begin{array}{c}\log (n) \\
\text { both }\end{array}$ & $\begin{array}{c}\log (N) \\
\text { both }\end{array}$ & $\begin{array}{c}\chi^{2} \\
\text { both }\end{array}$ \\
\hline $32.8+0.20 \mathrm{~A}$ & $\mathrm{c}$ & - & - & - & $\mathrm{g}$ & $6.14 \pm 0.34$ & $13.48 \pm 0.23$ & 0.00 & - & - & - & - \\
\hline W44 & $\mathrm{c}, \mathrm{sa}$ & - & - & - & $\mathrm{f}$ & $6.21 \pm 0.19$ & $14.33 \pm 0.64$ & 0.16 & - & - & - & - \\
\hline W51 W & $\mathrm{c}$ & - & - & - & $\mathrm{g}$ & $5.95 \pm 0.25$ & $13.34 \pm 0.23$ & 0.00 & - & - & - & - \\
\hline W51 N C2 & $\mathrm{c}$ & - & - & - & $\mathrm{f}$ & $6.55 \pm 0.28$ & $14.00 \pm 0.41$ & 1.18 & - & - & - & - \\
\hline K3-50 & $\mathrm{c}$ & - & - & - & e & $5.95 \pm 1.07$ & $13.18 \pm 1.18$ & 0.00 & - & - & - & - \\
\hline ON 3 & $\mathrm{c}, \mathrm{sa}$ & - & - & - & $\mathrm{e}$ & $5.41 \pm 0.74$ & $13.07 \pm 0.32$ & 0.00 & - & - & - & - \\
\hline ON $2 \mathrm{~S}$ & $\mathrm{c}$ & - & - & - & $\mathrm{g}$ & $6.18 \pm 0.60$ & $13.43 \pm 0.28$ & 0.00 & - & - & - & - \\
\hline DR21 S & $\mathrm{c}$ & - & - & - & $\mathrm{g}$ & $5.98 \pm 0.27$ & $13.58 \pm 0.30$ & 0.00 & - & - & - & - \\
\hline $\mathrm{W} 75(\mathrm{OH})$ & $\mathrm{c}, \mathrm{sa}$ & - & - & - & $\mathrm{f}$ & $6.17 \pm 0.15$ & $14.02 \pm 0.33$ & 0.16 & - & - & - & - \\
\hline $\mathrm{W} 75 \mathrm{~N}$ & $\mathrm{c}$ & - & - & - & $\mathrm{f}$ & $6.24 \pm 0.22$ & $13.62 \pm 0.40$ & 1.66 & - & - & - & - \\
\hline S157 & $\mathrm{c}$ & - & - & - & e & $7.30 \pm 2.64$ & $13.09 \pm 1.05$ & 0.55 & - & - & - & - \\
\hline S158 & $\mathrm{c}$ & - & - & - & $d$ & $6.08 \pm 0.23$ & $13.80 \pm 0.02$ & 0.28 & - & - & - & - \\
\hline S158 A & $\mathrm{c}$ & - & - & - & $\mathrm{h}$ & $6.22 \pm 0.19$ & $13.54 \pm 0.03$ & 0.61 & - & - & - & - \\
\hline IRAS $00338+6312$ & $\mathrm{c}, \mathrm{sa}$ & - & - & - & d & $6.03 \pm 0.20$ & $13.22 \pm 0.04$ & 0.00 & - & - & - & - \\
\hline NGC 281 & $\mathrm{c}$ & - & - & - & $\mathrm{d}$ & $5.87 \pm 0.15$ & $13.22 \pm 0.15$ & 1.69 & - & - & - & - \\
\hline W3(2) & $\mathrm{c}, \mathrm{sa}$ & - & - & - & $\mathrm{g}$ & $6.26 \pm 0.33$ & $13.16 \pm 0.02$ & 0.00 & - & - & - & - \\
\hline $\mathrm{W} 3(\mathrm{OH})$ & $\mathrm{c}, \mathrm{sa}$ & - & - & - & $\mathrm{g}$ & $6.80 \pm 0.92$ & $14.16 \pm 0.35$ & 0.00 & - & - & - & - \\
\hline IRAS $02461+6147$ & $\mathrm{c}$ & - & - & - & $\mathrm{e}$ & $7.15 \pm 2.59$ & $12.80 \pm 0.90$ & 3.45 & - & - & - & - \\
\hline $\mathrm{S} 235$ & $\mathrm{c}$ & - & - & - & $d$ & $5.71 \pm 0.17$ & $13.45 \pm 0.13$ & 0.03 & - & - & - & - \\
\hline $\mathrm{S} 255 / 7$ & $\mathrm{c}$ & - & - & - & $\mathrm{d}$ & $5.62 \pm 0.17$ & $13.54 \pm 0.12$ & 0.02 & - & - & - & - \\
\hline MON R2 & $\mathrm{c}$ & - & - & - & $\mathrm{e}$ & $5.67 \pm 0.53$ & $12.79 \pm 0.01$ & 0.01 & - & - & - & - \\
\hline MON R2(IRS3) & $\mathrm{c}$ & - & - & - & $\mathrm{d}$ & $5.95 \pm 0.27$ & $13.00 \pm 0.06$ & 0.46 & - & - & - & - \\
\hline
\end{tabular}

a - Fits to $\mathrm{CS} \mathrm{J}=2 \rightarrow 1,3 \rightarrow 2,5 \rightarrow 4, \& 7 \rightarrow 6$. b - Fits to CS J $=2 \rightarrow 1,3 \rightarrow 2, \& 5 \rightarrow 4$. c - Data from June 1990 . d - Fits to C $\mathrm{C}^{34} \mathrm{~S} \mathrm{~J}=2 \rightarrow 1,3 \rightarrow 2, \quad \& 5 \rightarrow 4$. e - Fits to $\mathrm{C}^{34} \mathrm{~S} \mathrm{~J}=2 \rightarrow 1, \& 3 \rightarrow 2$. f - Fits to $\mathrm{C}^{34} \mathrm{~S} \mathrm{~J}=3 \rightarrow 2,5 \rightarrow 4, \& 7 \rightarrow 6$. g - Fits to $\mathrm{C}^{34} \mathrm{~S} \mathrm{~J}=3 \rightarrow 2, \& 5 \rightarrow 4$. h - Fits to $\mathrm{C}^{34} \mathrm{~S} \mathrm{~J}=2 \rightarrow 1,3 \rightarrow 2,5 \rightarrow 4, \& 7 \rightarrow 6 . \quad \mathrm{i}-\mathrm{Fits}$ to all $4 \mathrm{CS}$ transitions and $\mathrm{C}^{34} \mathrm{~S} \mathrm{~J}=2 \rightarrow 1,3 \rightarrow 2, \& 5 \rightarrow 4 . \quad \mathrm{j}-$ Fits to all $4 \mathrm{CS}$ transitions and $\mathrm{C}^{34} \mathrm{~S} \mathrm{~J}=2 \rightarrow 1, \& 3 \rightarrow 2$. $\mathrm{k}-$ Fits to all $4 \mathrm{CS}$ transitions and $\mathrm{C}^{34} \mathrm{~S} \mathrm{~J}=2 \rightarrow 1$. $1-$ Fits to $\mathrm{CS} \mathrm{J}=2 \rightarrow 1,3 \rightarrow 2, \& 5 \rightarrow 4$ and $\mathrm{C}^{34} \mathrm{~S} \mathrm{~J}=2 \rightarrow 1$. sa - Self absorbed source. 